\title{
Paleovolcanic reconstruction in the Tokaj Mountains
}

\author{
Tibor Zelenka \\ Institute of Mineralogy and Geology, \\ University of Miskolc, Miskolc
}

\author{
Pál Gyarmati \\ Geological Institute of Hungary, Budapest
}

\section{János Kiss}

Eötvös Loránd Geophysical Institute of Hungary, Budapest

\begin{abstract}
The Tokaj Mts, situated in the northeastern part of the inner arc of the Carpathians, forms a part of a Miocene calc-alkaline andesitic-dacitic-rhyolitic volcanic island arc. The ancient volcanic structures were reconstructed on the basis of the 1:50 000-scale and 22 sheets of the 1:25 000-scale geologicpetrologic maps, as well as the revision of the volcanic facies in 150 boreholes. Multispectral and SAR satellite imagery, aerial photos, data and maps of airborne geophysical surveys (magnetic and radiometric), gravity-filtered anomaly maps, geochemical (soil and stream sediment $\mathrm{Au}, \mathrm{As}, \mathrm{Sb}, \mathrm{Hg}$ ) concentration distribution maps and the K/Ar dating of 132 samples from 80 paleomagnetic measurements were also used.

The anomalies were only taken into consideration in the interpretation if the coincident results of at least 3 methods indicated the presence of any volcanic structure. In consequence, 91 map-scale volcanic structures were identified by morphology - complex calderas, single lava domes, volcanic fissures, subvolcanic intrusions, diatremes, stratovolcanoes and postvolcanic formations. Conclusions were also drawn regarding the link to the volcanic structures and prospective occurrences of the mineral resources of the Tokaj Mts: andesite, dacite, welded zeolitic tuff, K-metasomatite, perlite, pitchstone, pumice, bentonitic, illitic, kaolinitic, diatom-bearing and silicified lacustrine sediments, hydrothermal $\mathrm{Au}-\mathrm{Ag}$ and $\mathrm{Pb}-\mathrm{Zn}$ veins, and $\mathrm{Hg}$ stockwerks.
\end{abstract}

Key words: exploration methods, volcanoes, island arc, calc-alkaline, morphology, complex interpretation

\section{Introduction}

The subject matter was developed under the leadership of Tibor Zelenka by Pál Gyarmati, János Kiss, László Vértesy, István Horváth, Zoltán Pécskay and Emó Szalay, and was supported by OTKA project T022769. The study aimed at

\footnotetext{
Addresses: T. Zelenka: H-3515 Miskolc-Egyetemváros, Hungary, e-mail: tibor.zelenka@gmail.com P. Gyarmati: H-1143 Budapest, Stefánia út 14, Hungary J. Kiss: H-1145 Budapest, Kolumbusz u. 17-23, Hungary

Received: March 13, 2012; accepted: April 14, 2012
} 
establishing an up-to-date approach to the paleovolcanic reconstruction of the Tokaj Mts based on geologic, volcanological, geophysical, geochemical and geomorphologic data.

\section{Exploration history}

Reviewing the publications on volcanology concerning the Tokaj Mts from the last 250 years, one can find remarkable statements from almost every author.

The essays of Beudant (1822), Richthofen (1861) and Wolf (1869) are the pioneers of the recognition of geologic formations. The fundamental monograph of Szabó (1867) entitled "Tokaj-Hegyalja és környékének földtani viszonyai" (Geologic features of Tokaj-Hegyalja and its surroundings) provided the basis for the recognition of the volcanic structure of the mountains. Szádeczky (1897) was the first to recognize that the amphibole andesite had broken through the rhyolite.

Pálfy (1927) studied the link between volcanism and ore formation. Rozlozsnik (1937) described rhyolite domes around Mád. The observations of Telegdi Róth (1935) are particularly important: according to him, at Szegi "the andesite rises transected the younger rhyolite tuff series". The monograph on the Szerencs Hills by Hoffer $(1928,1937)$ is an essay with a volcanological approach, meeting contemporary demands. Liffa (1940) described Tó Hill at Boldogkóváralja as a pyroxene andesite stratovolcano.

Intensive mineral resource prospecting in the middle of the past century also favored basic exploration. Lengyel (1959) specified in several publications the mineralogical and petrologic description of the andesite varieties. Zelenka (1964) recognized five acidic eruption phases in the Szerencs Embayment.

Székyné Fux (1970) linked the formation of the Telkibánya ores to a subvolcanic potassic trachyte body in her genetic model. In our recent view this is a subvolcanic andesite body subjected to potassic metasomatism. The statements of Pantó (1963) have a particular volcanological importance concerning ignimbrite genetics and the distinction between plutonic and volcanic facies (Pantó 1967). Jugovics (1962) recognized several undescribed subvolcanic bodies (Tállya, Erdóbénye) and an eruption center (Tarcal).

The modern geologic mapping of the Tokaj Mts was carried out in the period of 1959 to 1972, resulting in the "Geologic Map of the Tokaj Mts" (Gyarmati et al. 1977). The forerunner of the recent paleovolcanic reconstruction is the map entitled "Structural-volcanotectonic sketch of the Tokaj Mts" (Gyarmati 1977a), published as a supplement to the monograph "Intermediate volcanism in the Tokaj Mts" (Gyarmati 1977b). The author also published the schematic spatial and temporal connections of the volcanism in the "Tokaj Mts" chapter of the "Pannon Enciklopédia" (Pannonian Encyclopedia) (Gyarmati 1997).

The geochemical survey of the area was carried out through FinnishHungarian cooperation (Hartikainen et al. 1992, 1993). 
Remote sensing was used first at Telkibánya to detect a double andesite caldera structure with rhyolite domes within and andesite parasitic cones at the rim (Horváth et al. 1989). The smaller subsequent tuff volcano structure with its radial and concentric quartz veins was also recognized.

Having collected the geologic, volcanological, geophysical, geochemical data and morphologic analyses made up to then, the modern volcanological map of the Tokaj Mts was drawn in 2000 (Fig. 1) and 2007 (Zelenka 2000; Zelenka et al. 2007). The fluid inclusion studies on samples from the hydrothermal centers of the area (Molnár et al. 1995, 1999) contributed to the determination of the pressure-temperature conditions of the ore generating fluids. Recent studies indicated ancient caldera structures and subvolcanic bodies around every hydrothermal-postvolcanic field (Molnár et al. 1999, 2002).

Spectral depth estimations were made around the centers using gravity and airborne magnetic sections to estimate the thickness of the andesite cover and the supposed depth of the basement (Kiss and Prácser 2000).

\section{Geology of the Tokaj Mountains}

In our studies the data on the geology and history of the Tokaj Mountains were revised (Gyarmati and Zelenka 2000; Zelenka 2000), shown in Figure 2.

As far as geology is concerned, the mountains can be divided into a western and an eastern unit. The western unit extends from the Hernád Lineament to the Hercegkút and Hosszúrét Creeks, with Sarmatian and Pannonian volcanic rocks and sediments on the surface. Although the basement is still unexplored, it is assumed to consist of Paleozoic shale on the basis of inclusions in pyroclastics. The eastern unit extends to the Ronyva Creek. In this unit the Proterozoic Lower Paleozoic metamorphosed basement crops out in the north, while in the south a Mesozoic carbonate-dominated basement is overlain by Badenian volcanics and sediments.

The age of volcanism in the mountains ranges from 15 to 9 Ma BP, i.e. from Late Badenian to Early Pannonian, determined by K/Ar dating (Pécskay et al. 1986; Pécskay et al. 1995; Molnár and Pécskay 2002) and according to paleontological data (Fig. 2).

The character of the volcanism was calc-alkaline based on geochemical analyses of major elements (Fig. 3) and rare earth elements (Fig. 4). The magma possibly originated from the lower crust and the upper mantle.

The volcanism over the basement, originally in NW-SE zones and later during the continuously diminishing depth of the archipelego, shows 3 phases. At first phreatomagmatic eruptions produced large masses of rhyolitic-dacitic pyroclastite during the Early Badenian. In the next phase submarine peperitic, hyaloclastic andesite, andesite lava beds and stratovolcanic andesite continued the succession (according to the Tállya-15 and Füzérkajata-2 boreholes); 


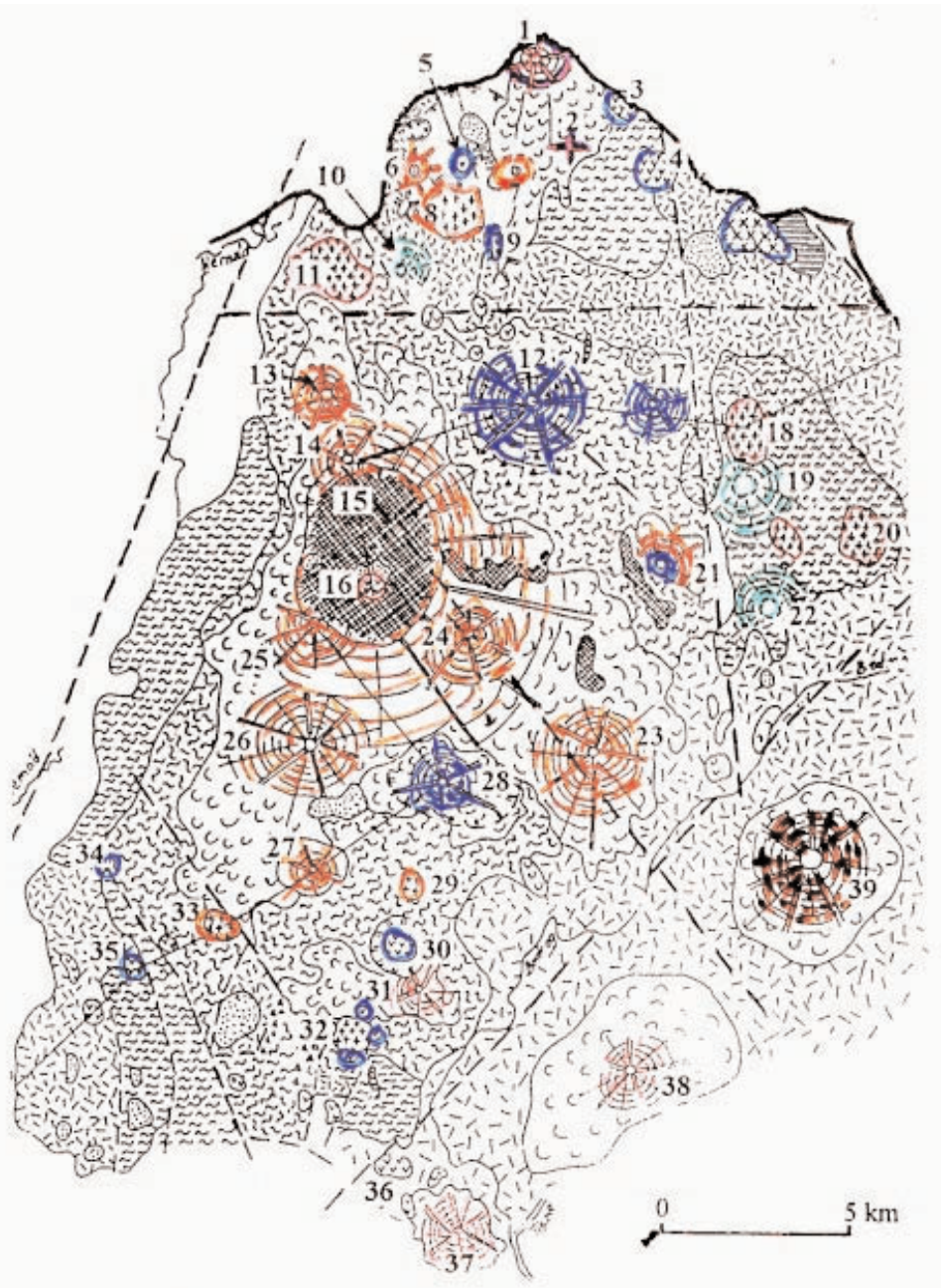

Fig. 1

Geologic-volcanological paleovolcanic reconstruction of 2000 (Gyarmati and Zelenka 2000). 1. Füzér, Remete Hill, dacite; 2. Füzér Castle, dacite; 3. Pusztafalu, Tolvaj Hill, rhyodacite; 4. Pusztafalu, Hársas, rhyodacite; 5. Hollóháza, Pál Hill, rhyolite; 6. Pányok, Nagy Hill, andesite; 7. Hollóháza, Május Hill, andesite; 8. Telkibánya, Kánya and Gyepü Hills, K-metasomatite; 9. Nyíri, Fehér Hill, rhyolite; 10. Telkibánya, Youth Camp, rhyolite tuff; 11. Gönc, Vas and Ör Hills, dacite; 12. Nagybózsva, Fekete Hill, rhyolite stratovolcano; 13. Gönc, Borsó Hill, andesite; 14. Hejce, Gergely Hill, upper laminar andesite; 15. Regéc, andesite caldera; 16. Regéc, Vár Hill, rhyodacite; 17. Kishuta - Pálháza, Som Hill, rhyolite; 18. Vágáshuta, Fekete Hill, dacite subvolcano; 19. Vágáshuta, Nyúl Spring, rhyolite tuff; 20. Sátoraljaújhely, Sátor Hill, dacite subvolcano; 21. Makkoshotyka, Katuska, andesite - rhyolite; 22. Sárospatak, Király Hill, rhyolite tuff; 23. Tolcsva, Fekete Hill, andesite; 24. Óhuta, Zabarla - Hajagos, andesite; 25. Arka, Amgoska, andesite; 26. Baskó, Nagy-Korsós, andesite; 27. Erdőbénye, Szokolya, olivine andesite; 28. Erdőhorváti, Szokolya, Nagy-Páca, rhyolite; 29. Erdóbénye, Mulató Hill, dacite subvolcano; 30. Erdőbénye, Spa, rhyolite; 31. Szegi, Cigány Hill, dacite; 32. Mád, Diós, andesite and rhyolite; 33. Tállya, Kopasz, andesite subvolcano; 34. Abaújszántó, Sátor - Krakkó Hill, rhyolite; 35. Golop, Somos, rhyolite; 36. Tarcal, Terézia Chapel, rhyolite; 37. Tokaj, Nagy Hill, dacite; 38. Zalkod, covered andesite, dacite; 39. Apróhomok, olivine basalt 

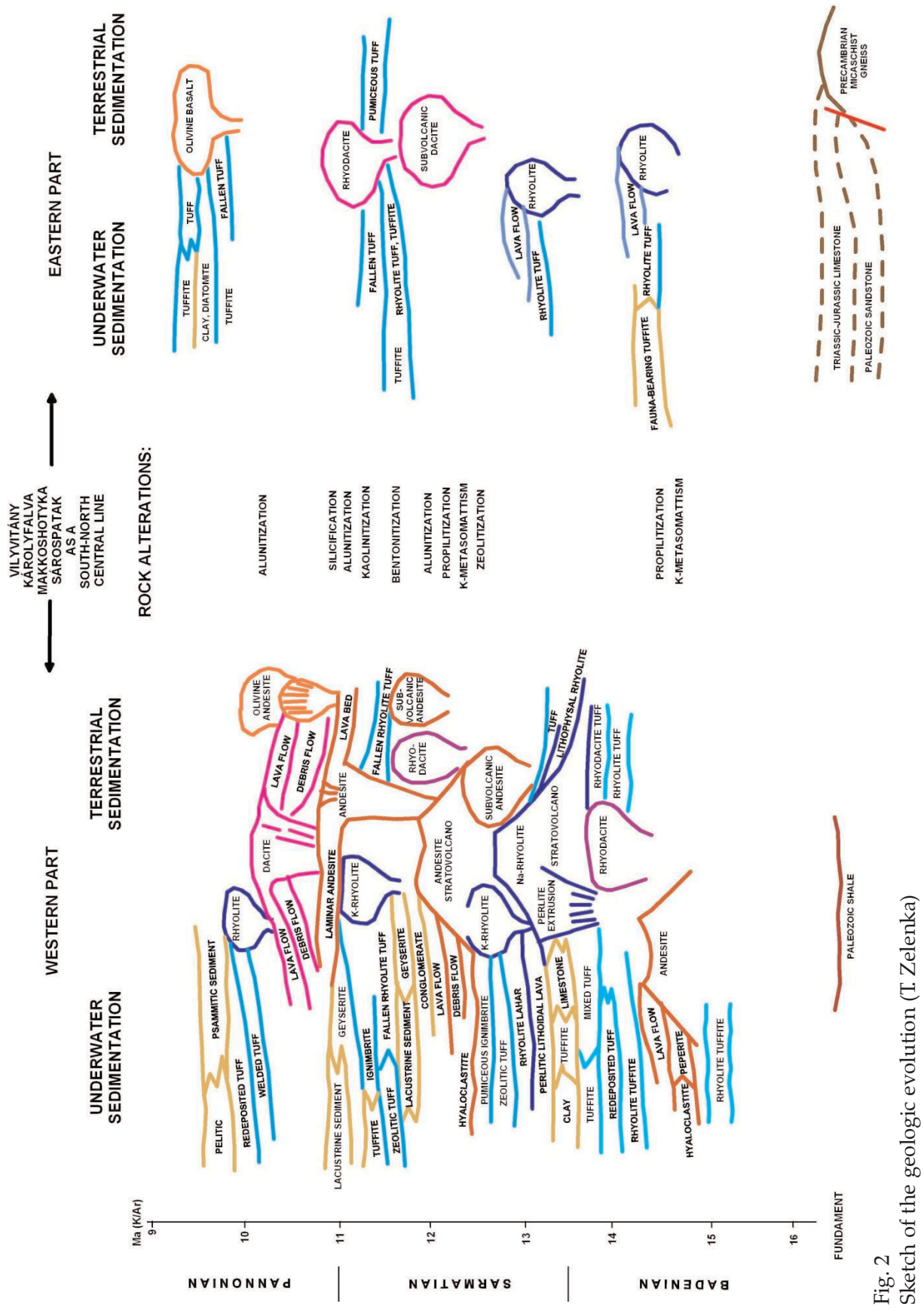

Central European Geology 55, 2012 


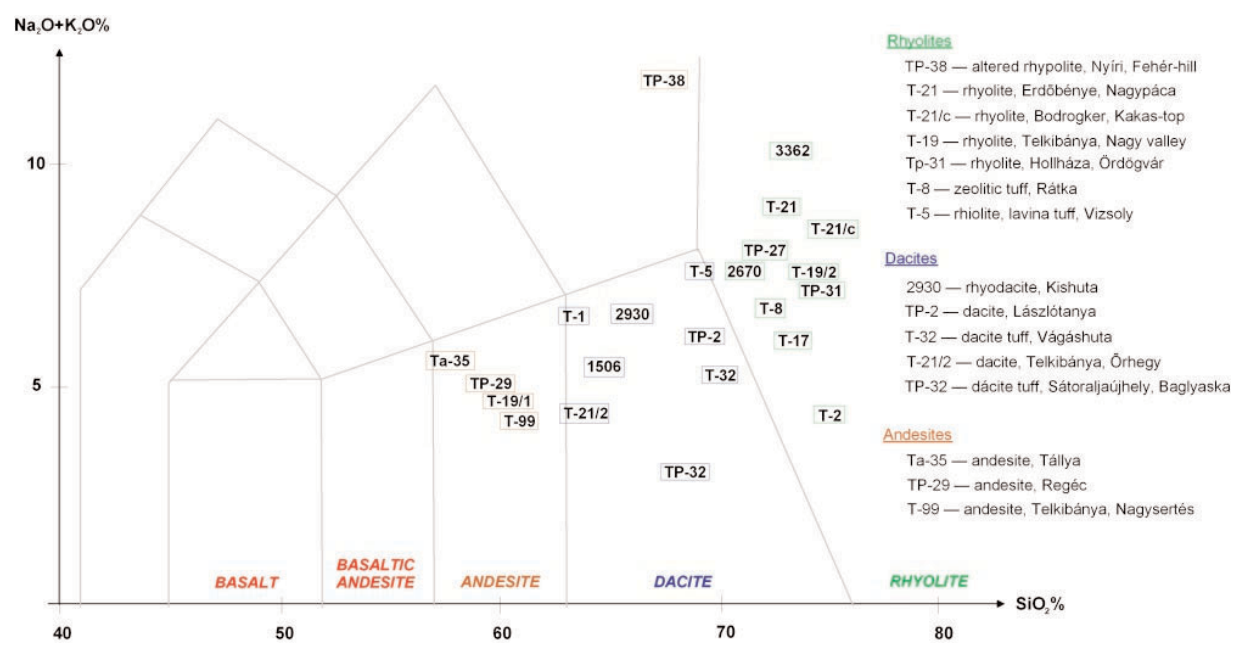

Fig. 3

TAS diagram of the Tokaj Mts volcanic rocks (P. Gyarmati)

thereafter the volcanic cycle was closed by dacitic subvolcanic intrusions (Sátoraljaújhely-8, Kishuta-1, Baskó-3 boreholes).

The phreatomagmatic eruptions, contemporaneously with transgression in the Early Sarmatian stage, produced large volumes of rhyolitic ignimbrite flows and fallen pyroclastics with small lava domes (Erdőhorváti-Szokolya-Nagypáca, Fekete Hill at Kishuta). Several stratovolcanic eruption centers were formed in the central part of the mountains (Hollóháza, Regéc, Mád), producing large masses of andesite and pyroclastics.

The subvolcanic andesite and dacite bodies of this volcanic phase were partly affected by potassic metasomatism (Telkibánya, Óhuta, Sárospatak, Mád). Simultaneous postvolcanic hydrothermal activity produced precious metal ore veins and clay deposits in lacustrine successions (Rátka, Hollóháza, Füzérradvány, Erdőbénye).

At the Sarmatian-Pannonian boundary ignimbritic and ash-flow tuff originated from several minor rhyolitic centers (Vizsoly, Abaújszántó), bound to $\mathrm{N}-\mathrm{S}$ striking tectonic zones. In the last phase of volcanism mainly dacite monovolcanoes with lava and debris flows were formed (Nagy Hill at Tokaj, Cigány Hill at Szegi). Olivine andesite domes, dykes (Erdőbénye, Szokolya) and an olivine basalt diatreme (Sárospatak, Apróhomok-10 borehole) indicate the final calc-alkaline volcanic activity. 


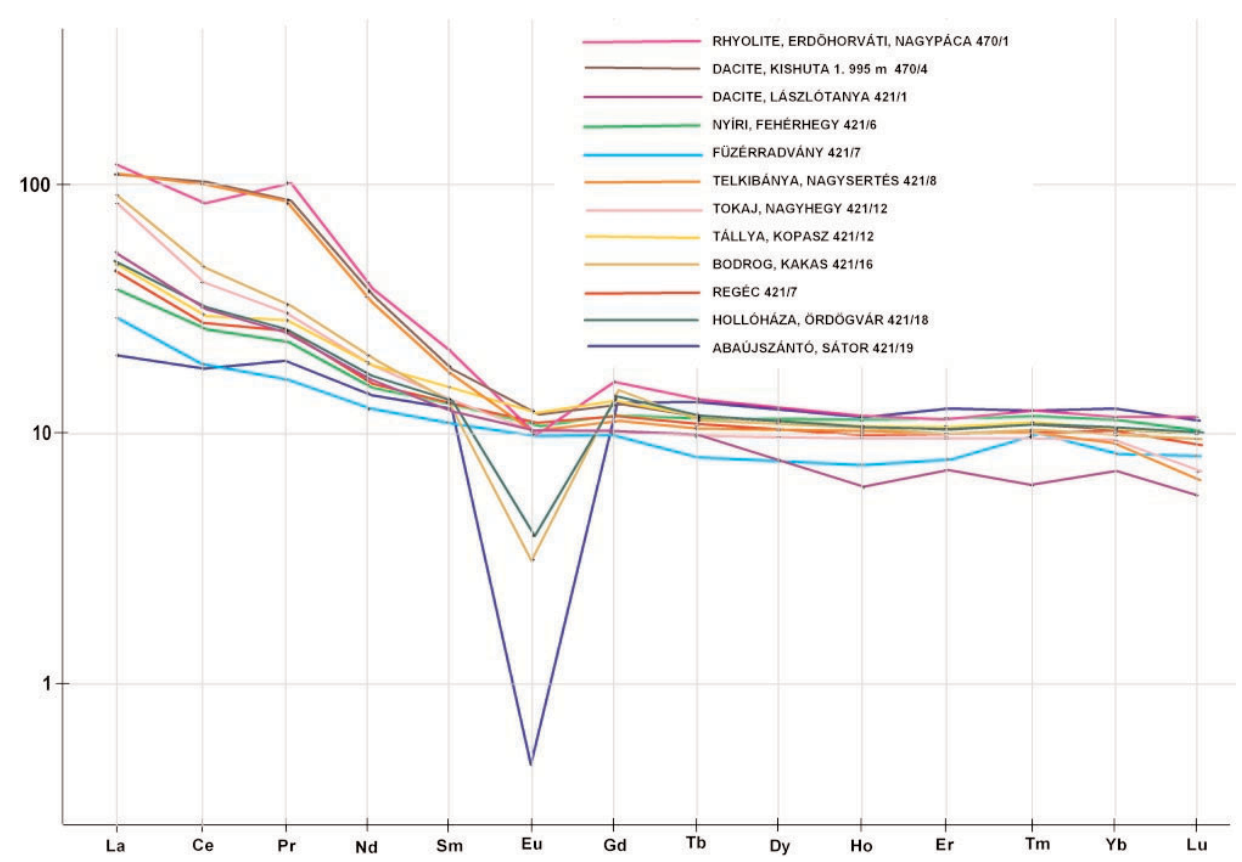

Fig. 4

Rare earth element contents of Tokaj Mts volcanic rocks normalized to chondrite (P. Gyarmati)

\section{Applied exploration methods}

Geologic-volcanological analysis

The 1:25 000-scale geologic map series drawn in the Geological Institute of Hungary between 1959 and 1972 under Gábor Pantó's direction, the summarized 1:50 000-scale geologic map depicting the entire Tokaj Mts (Gyarmati et al. 1972) and the above-mentioned tectonic map (Gyarmati 1977a) served as basis of our work.

The geologic work began with revisiting the sites and reviewing the logs of the major boreholes (667 units of mapping or structural exploration and several hundred mineral resource exploration holes). The logs were reinterpreted; the characteristic volcanic facies were identified. In the most important 150 boreholes the facies, the morphology, the genetics and the mutual relationship of the lava flows and pyroclastics were specified. According to geomorphologic observations the erosion of an at least 200-300 m thick section was considered from the end (?) of the Sarmatian, over the area of the entire Tokaj Mts. 
Based on the original geologic map and the volcanological field observations in certain areas the modern paleovolcanic map of the Tokaj Mountains was compiled, indicating 39 supposed volcanic eruption centers (Fig. 1).

Morphological analysis based on remote sensing

The morphological criteria applied for identifying volcanic structures were based on the work of Cas and Wright (1988). These analyses were principally aimed at detecting the erosional morphological features, but the major volcanic and tectonic structures were also indicated (Zelenka 1997, 1998, 2000).

The seven channels of Landsat TM-5, the panchromatic Spot- 1 scenes, and the data of previous airborne magnetic and radiometric surveys digitized by ELGI (Eötvös Loránd Geophysical Institute) provided the basis of an integrated volcanological interpretation (Kiss and Gulyás 1998; Gulyás et al. 2000).

The ancient ring-shaped escarpments of the tuff volcano at the Telkibánya shooting range, with the radial and concentric quartz vein outcrops, were identified (Horváth et al. 1989; Zelenka 2000). The ancient lava flows, pyroclastics, lava domes and the silicified and argillaceous sediments of the postvolcanic lake basins are well identifiable by stereo aerial photography. The eroded paleoshapes are also well recognizable on digital surface models made using SRTM data.

Based on satellite imagery the andesite calderas, the parasitic cones on their edges, the small rhyolite domes and the subvolcanic bodies within the calderas were reconstructed (Fig. 5). Landsat TM-5 2, 4 and 5 multispectral channel combinations and 5 and 7 single channel scenes proved to be the most appropriate for indicating eroded volcanic structures and volcanological interpretation.

\section{Geophysical data interpretation}

In the case of the Tokaj Mts gravimetry method plays an important role in the exploration of basement structures and the overlying high-density lava formations. Magnetic data specifically indicate the position of magnetite-bearing basic volcanic formations, even at some kilometers of penetration depth.

Radiometry (gamma-spectrometry) is limited to the exploration of the top layers to some $10 \mathrm{~cm}$ depth and to the indication of secondary alterations; it only indicates the underlying formations in the case of autochthonous soils.

1) Aeromagnetic map

The distribution of andesite and andesitodacite can be estimated from the data of airborne magnetic measurements. This parameter gives the best resolution among geophysical methods because of the variable susceptibility and geometry of the magnetite-bearing rocks. 


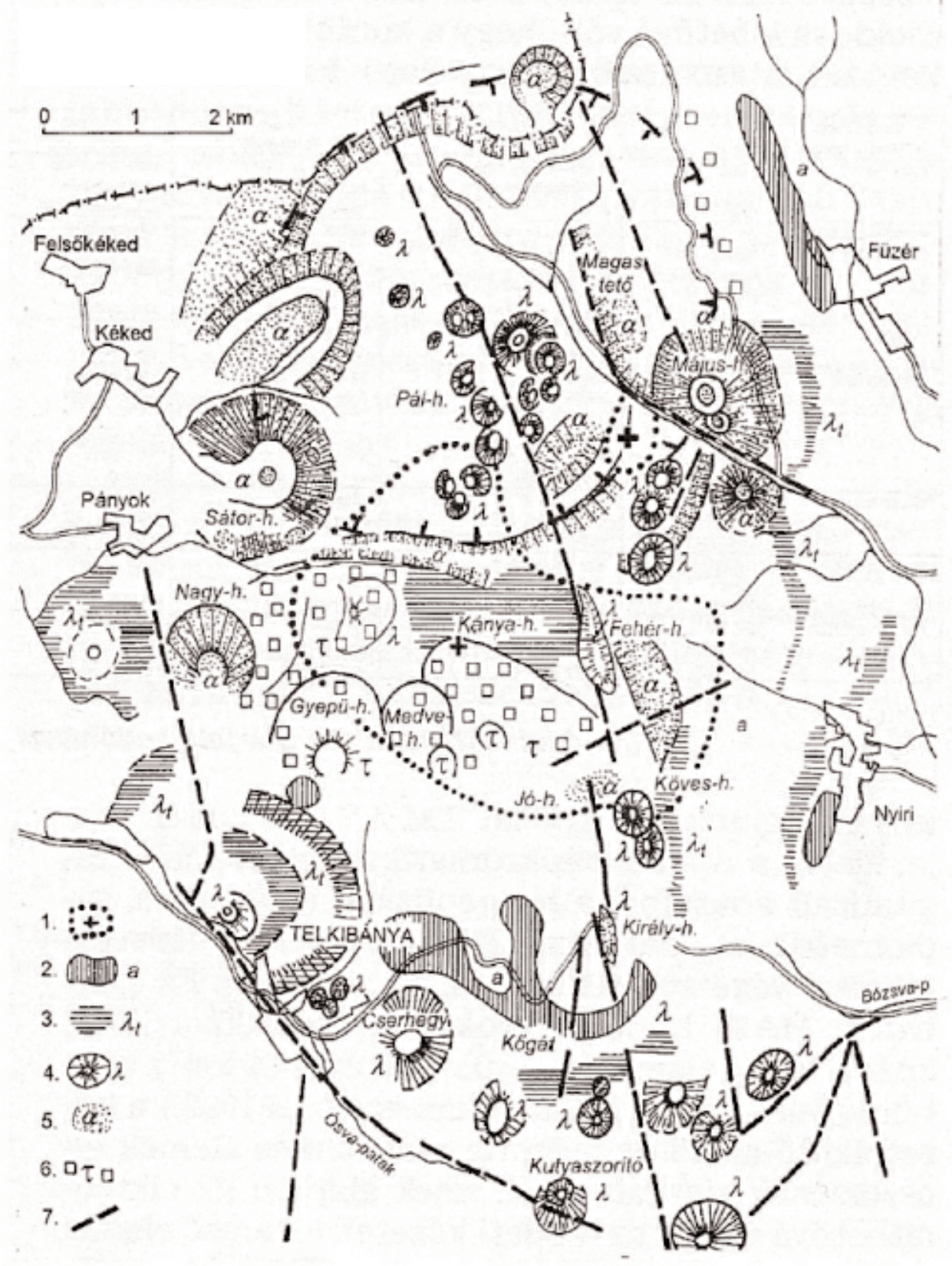

Fig. 5

Andesite caldera, subvolcanic body and rhyolite domes in the vicinity of Telkibánya (T. Zelenka). 1. gravity maximum; 2 . sediment; 3 . rhyolite tuff; 4 . rhyolite dome; 5 . andesite; 6 . K-metasomatite; 7. tectonic lineament 
2) Magnetic total gradient map

The nearest source bodies to the magnetic sensor cause the largest frequency and amplitude anomalies, with the highest gradients. The total magnetic gradient (or analytical signal) is appropriate for the demonstration of these changes (Fig. 6).

The value of the total magnetic gradient is high over outcropping volcanics. The anomaly pattern is very complicated, showing the inner complexity of the volcanic formations. In the case of covered lava formations the total gradient anomalies outline the lava bodies.

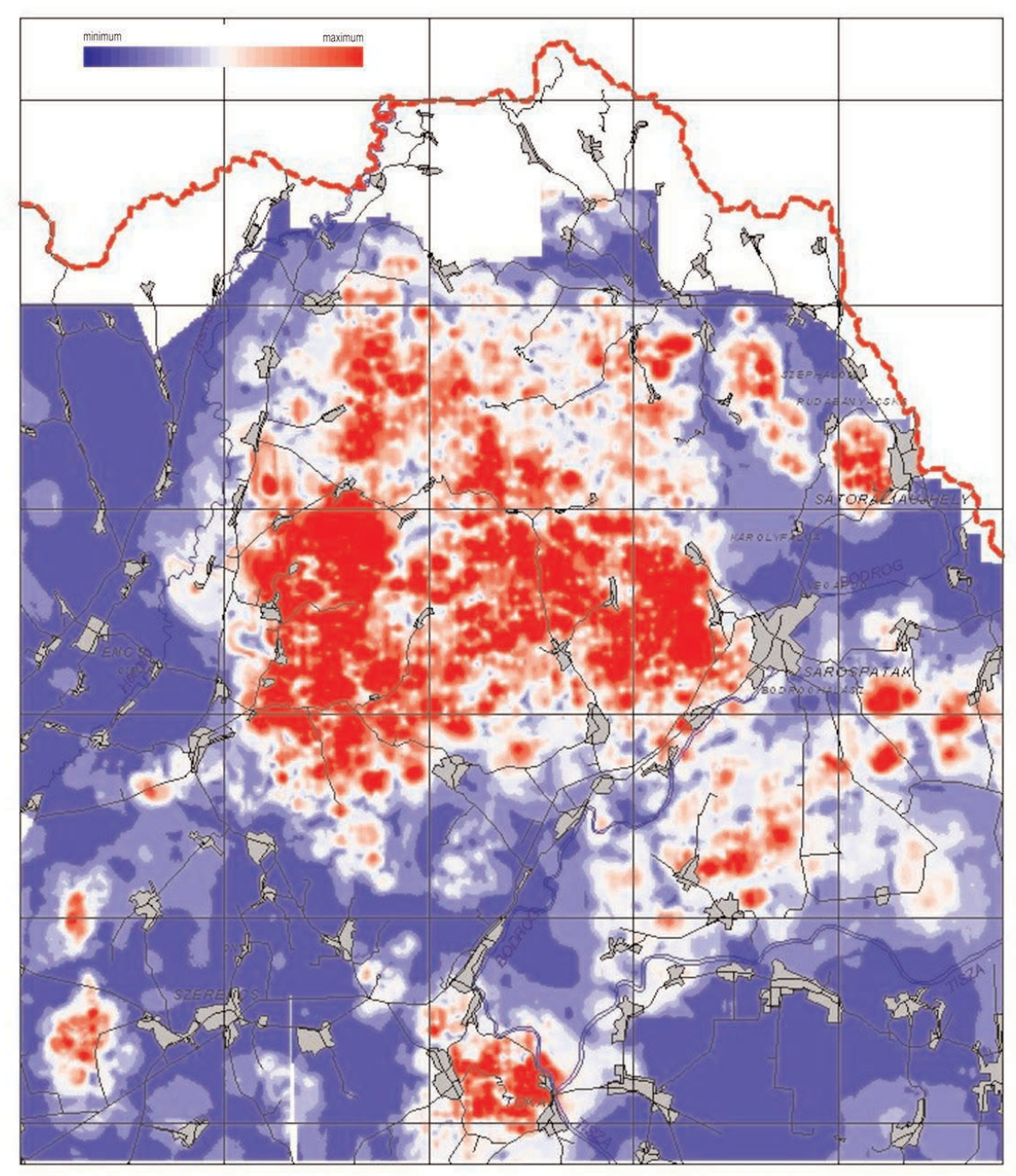

Fig. 6

Total magnetic gradient map from the data of the airborne survey (J. Kiss) 
3) Bouguer gravity anomaly map

One of the most apparent gravity anomalies of the area is the large northern gravity minimum, but its origin was not clarified by our interpretation. The pattern of the Bouguer gravity anomaly map (with a reduction density of 2000 $\mathrm{kg} / \mathrm{m}^{3}$ ) is basically correlated with the basement surface, but the effect of volcanics strongly influenced the gravity field due to the overlapping of the density ranges of volcanic and basement formations. The largest paleovolcanic eruption centers are well recognizable on the Bouguer anomaly map, appearing as gravity maxima.

4) Filtered Bouguer anomaly map

Some eruption centers in the area of the large gravity minimum, which are easy to find at surface or also on satellite scenes, cannot be seen on the original Bouguer anomaly map. The low-frequency effects of deep sources can be filtered out from the Bouguer anomaly map using digital data processing (high-pass filters with 15-30 km wavelength, 1000-1300 m penetration depth), and the effect of the shallow high-density volcanic formations, like andesite and rhyolite, appears (Fig. 7).

The process of edge detection was based on the frequency-filtered Bouguer anomaly maps. The map representation of the results of high-pass filtered data provides a lineament map showing the rock bodies of upper volcanic series, while the low-pass filtered map may give important interpretational help in the construction of the structural geologic map (representing the basement and the volcanic root zones) of the area.

5) Airborne radiometric map (Th, K, U)

The maximum values in the airborne thorium distribution map are apparently associated with the rhyolite ignimbrites, or lava domes (Fig. 8), and these are typical for the Szerencs-Mád ignimbrite and Erdőhorváti rhyolite tuffs as well, which fill in the inner part of rhyolite calderas. The uranium maximum values can be found in the area of rhyolitic hydrothermal volcanic centers.

The largest maximum values (5-8\%) of potassium indicate K-metasomatism or alunitization, characteristic for the alterations of subvolcanic andesite rock bodies and for the zones of postvolcanic activity (Telkibánya, Óhuta, Sátoraljaújhely, Mád, Regéc, Szerencs).

6) Seismic measurements

The reason for the scarce availability of seismic data in the Tokaj Mts is the adverse geologic model. Seismic sections are restricted to the edges of the mountains, and these were recorded for exploration of the basement and supposed salt dome structures, so the seismic data are not very useful for paleovolcanic reconstruction. Practically, there was no seismic survey within the mountain range. The only short one, a $1200 \mathrm{~m}$-long reflection seismic section, was 


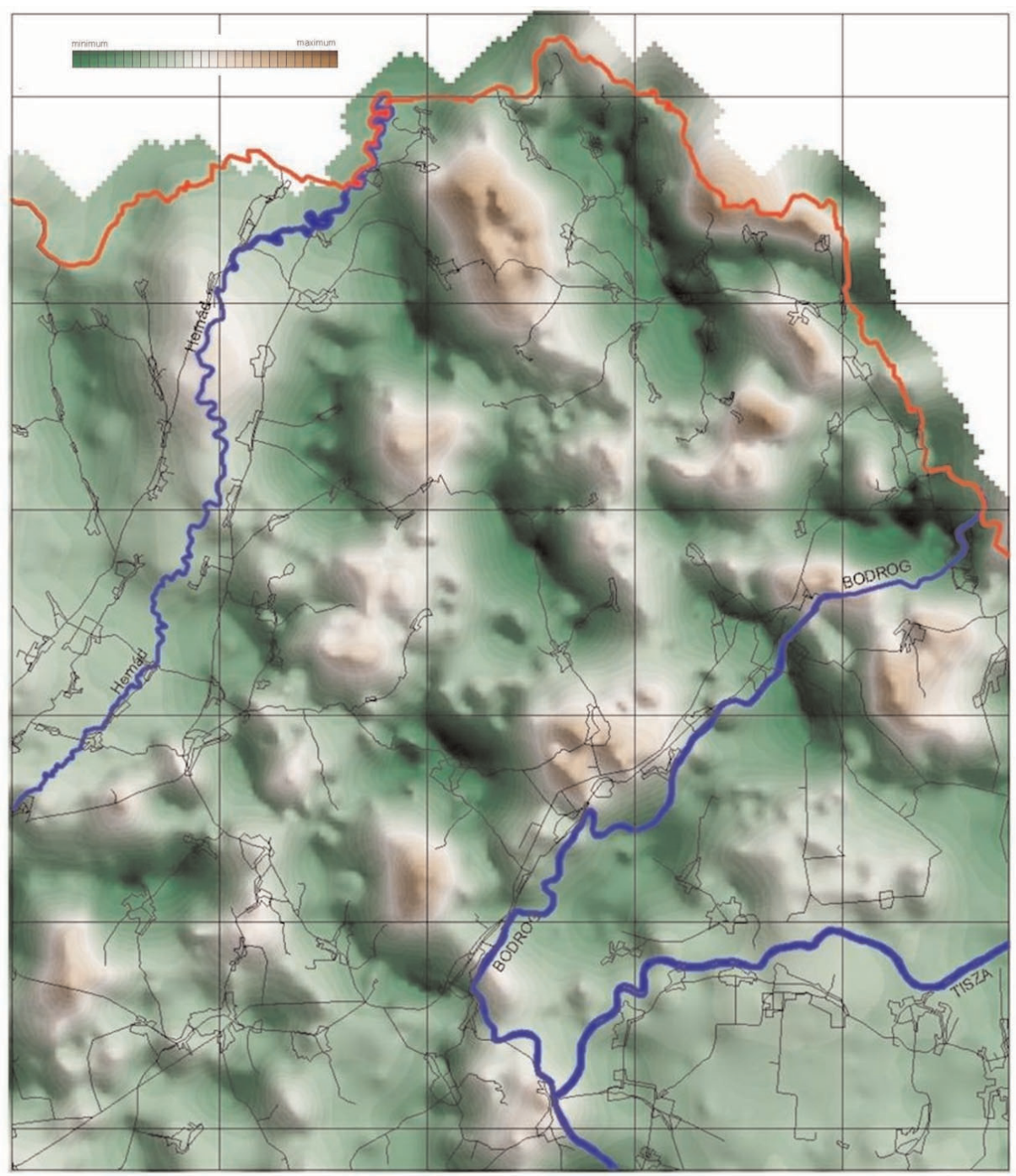

Fig. 7

Filtered Bouguer anomaly map (high-pass, $15 \mathrm{~km}$ ) with shading (J. Kiss)

recorded S of Mád to explore the thickness and deposition surfaces of volcanic (tuff flow) and marine sediments.

A deep seismic refraction tomography survey (CELEBRATION-2000 project) was carried out in Hungary, yielding a three-dimensional velocity data set. One of the seismic profiles (the CEL4) crossed the southwestern edge of the Tokaj Mts, partly revealing the root zone of the Szerencs caldera structure (there is an upper 
crust-like velocity anomaly at $8 \mathrm{~km}$ of depth, which is presumably caused by the remainders of a magma chamber; Hegedús et al. 2002).

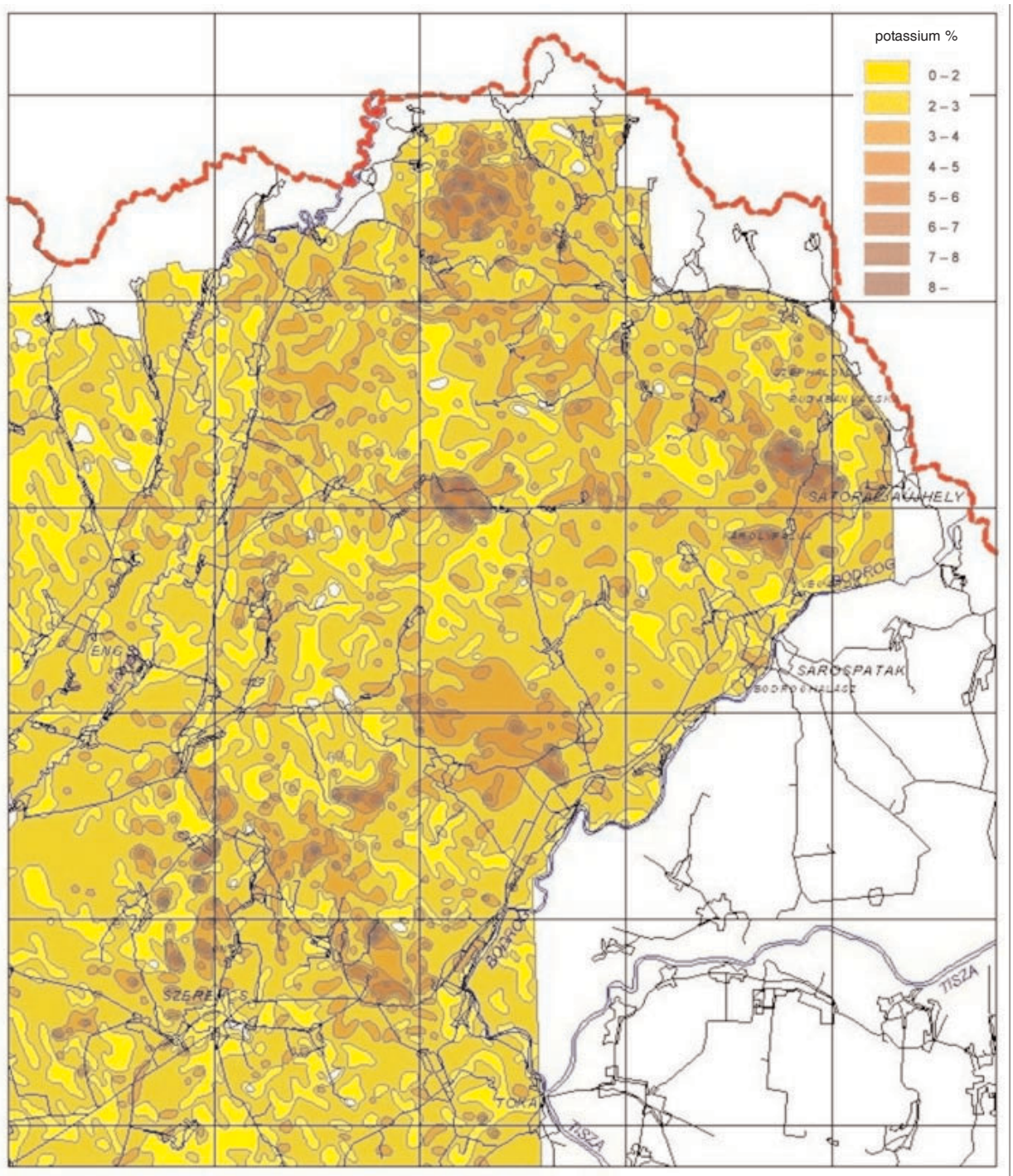

Fig. 8

Airborne radiometric potassium map (J. Kiss) 


\section{Geochemical surveys}

Stream sediment, soil and rock samples were taken from $0.7 \mathrm{~km}^{2}$ (on average) water discharge areas in the 1989-1992 period within the framework of a regional geochemical survey of the Hungarian Geological Institute.

Chemical analyses were made using the AAS method on nearly 690 stream sediment and more than 200 rock samples; here only the $\mathrm{K}, \mathrm{Na}$, Au, As, $\mathrm{Sb}$ and $\mathrm{Hg}$ data were considered. The primary aim was precious metal prospecting (Hartikainen et al. 1992, 1993). The secondary migration of the selected 6 elements is typically related to synmagmatic and postvolcanic hydrothermal processes. The Na distribution shows the composition of the volcanic formations in the mountains: the $0.5-2.0 \%$ range is characteristic for andesite, the $2.5-3.5 \%$ range for rhyolite. $\mathrm{K}$ content below $3 \%$ also characterizes andesite, in the range of 3-5\% acidic volcanites, while K-metasomatized rocks contain more than $5 \% \mathrm{~K}$.

The traces of postvolcanic activity in the volcanic formations can be found mainly at the ancient fumaroles, solfataras etc. The Au, Ag and accompanying As, $\mathrm{Sb}$ anomalies coincide with the eruption centers, $\mathrm{Hg}$ enrichments are at a distance from these. Complex $(\mathrm{Au}-\mathrm{As}-\mathrm{Sb}-\mathrm{Hg})$ anomalies are situated between or around eruption centers, or in areas subjected to K-metasomatism (Fig. 9).

\section{Paleomagnetic surveys}

Paleomagnetic surveys were carried out in the Tokaj Mts from 1970 (Fig. 10) on some outcrops of the major rock types (Nairn et al. 1971).

A systematic paleomagnetic survey began from the 1990s by Emóke MártonSzalay (Hungarian Geophysical Institute). From 1996, aiming to clear up the relationship between geologic, paleomagnetic and radiometric age data, specialists undertook common observations and sampling at outcrops of supposed eruption centers. At present more than 80 paleomagnetic measurements are available. The summarized interpretation of these, together with radiometric age determinations, is the topic of a separate paper (Szalay et al. 2007).

\section{Radiometric age determinations}

The K/Ar method was applied already from the 1970s by Kadosa Balogh (ATOMKI). The age data of the most important formations were determined by Zoltán Pécskay (ATOMKI) and his co-workers in the 1980s at the University of Debrecen, following the initiative of Vilma Székyné Fux. In the 1990s a coordinated sampling from outcrops and drill cores provided a large dataset. Up to the publication of this paper more than 132 individual $\mathrm{K} / \mathrm{Ar}$ method radiometric ages have been provided from the mountains, of which only the ones connected to eruption centers are considered here (Fig. 10). Detailed 
paleomagnetic and radiometric age data with geologic interpretation were prepared for publication (Szalay et al. 2007).

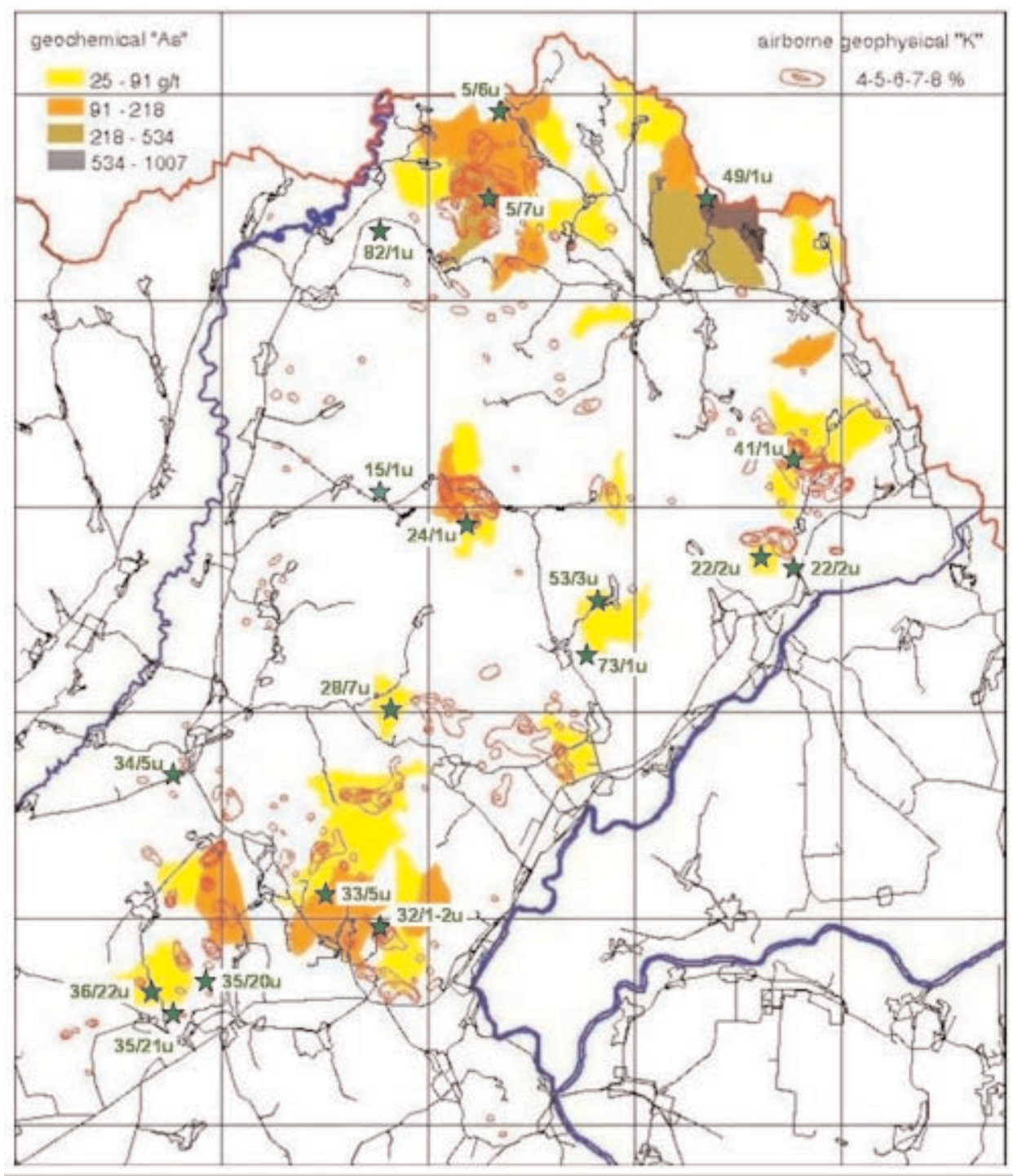

Fig. 9

Geochemical As (I. Horváth) and airborne radiometric K (J. Kiss) distributions with ore and other mineral resource occurrences in the areas of postvolcanic activity (T. Zelenka) 


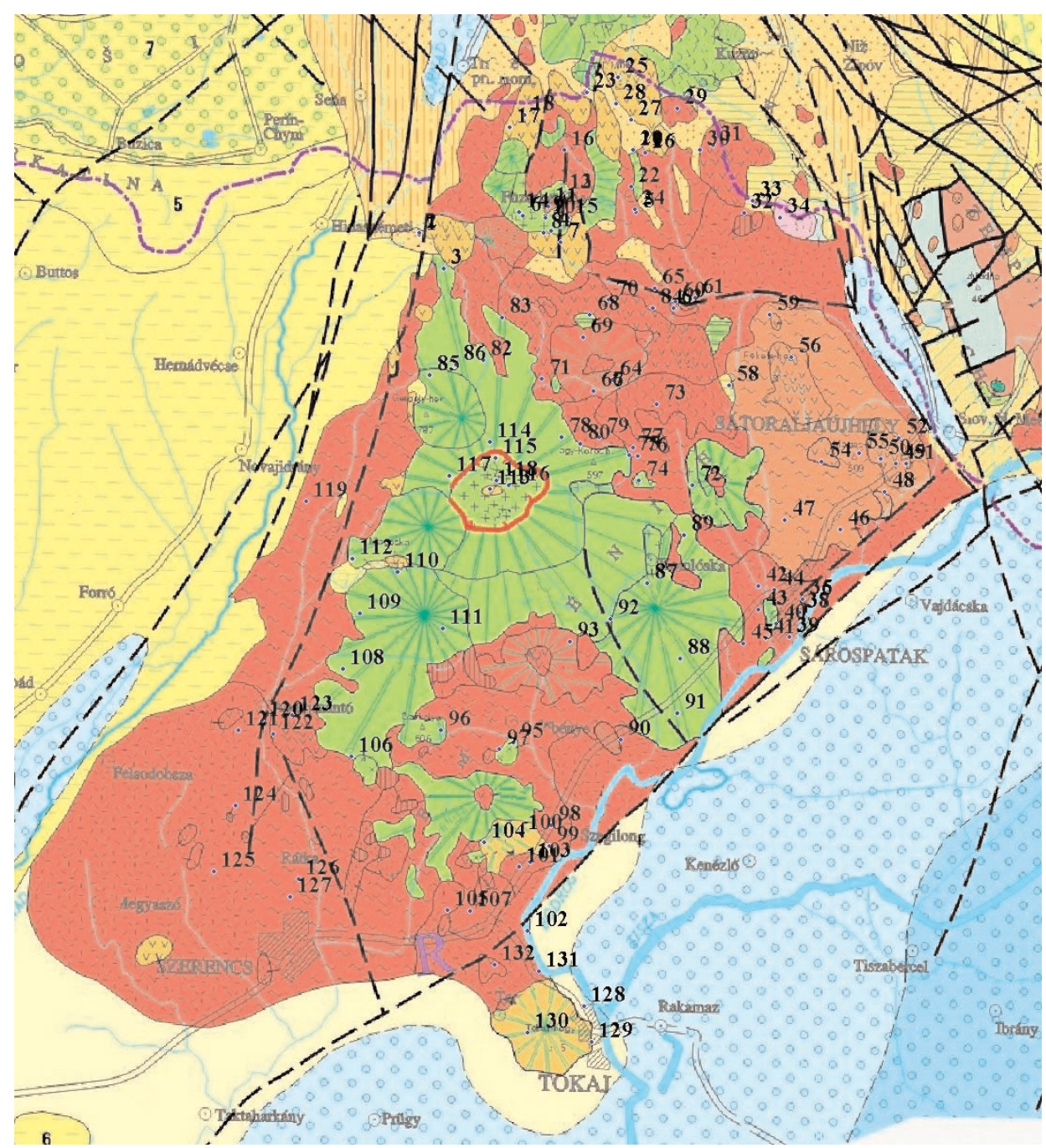

Fig. 10

Sites of paleomagnetic and K/Ar age sampling (Szalay et al. 2007)

\section{Identified volcanic structures of the Tokaj Mountains}

Beyond the methods above, our experience, observations and published data on recent volcanic areas were also utilized to identify of Tokaj volcanic structures. Detailed descriptions are given only for the most typical ones of these structures.

The parallel use of several exploration methods allowed us to deduce the eroded location and extension of the eruption centers, but the given datasets do 
not allow the full reconstruction of the volcanic structures. The data from boreholes in the mountains, which are more than $500 \mathrm{~m}$ deep (Ond-19, Mád-23, Tállya-15, Erdőhorváti-13, Baskó-3, Hidasnémeti-1, Telkibánya-2, Füzérkajata-2, Széphalom-1, Rudabányácska-2, Sátoraljaújhely-8, Sárospatak-3, Sárospatak-7), indicate that lava and pyroclastic product sequences of several eruptive phases can be followed up to 1-5 $\mathrm{km}$ from the supposed centers (Fig. 2).

The schematic maps (Figs 11 and 12) and Table 1 show the identification number of the sites and the rock names $(\mathrm{a}=$ andesite, $\mathrm{r}=$ rhyolite, $\mathrm{rd}=$ rhyodacite, $\mathrm{d}=$ dacite, $\mathrm{rf}=$ rhyolite tuff). The positions of the numbered volcanic centers are marked with a dot on the map. The maximum accuracy of the identification of the structures is $10 \mathrm{~m}$, according to the resolution of the applied satellite imagery.

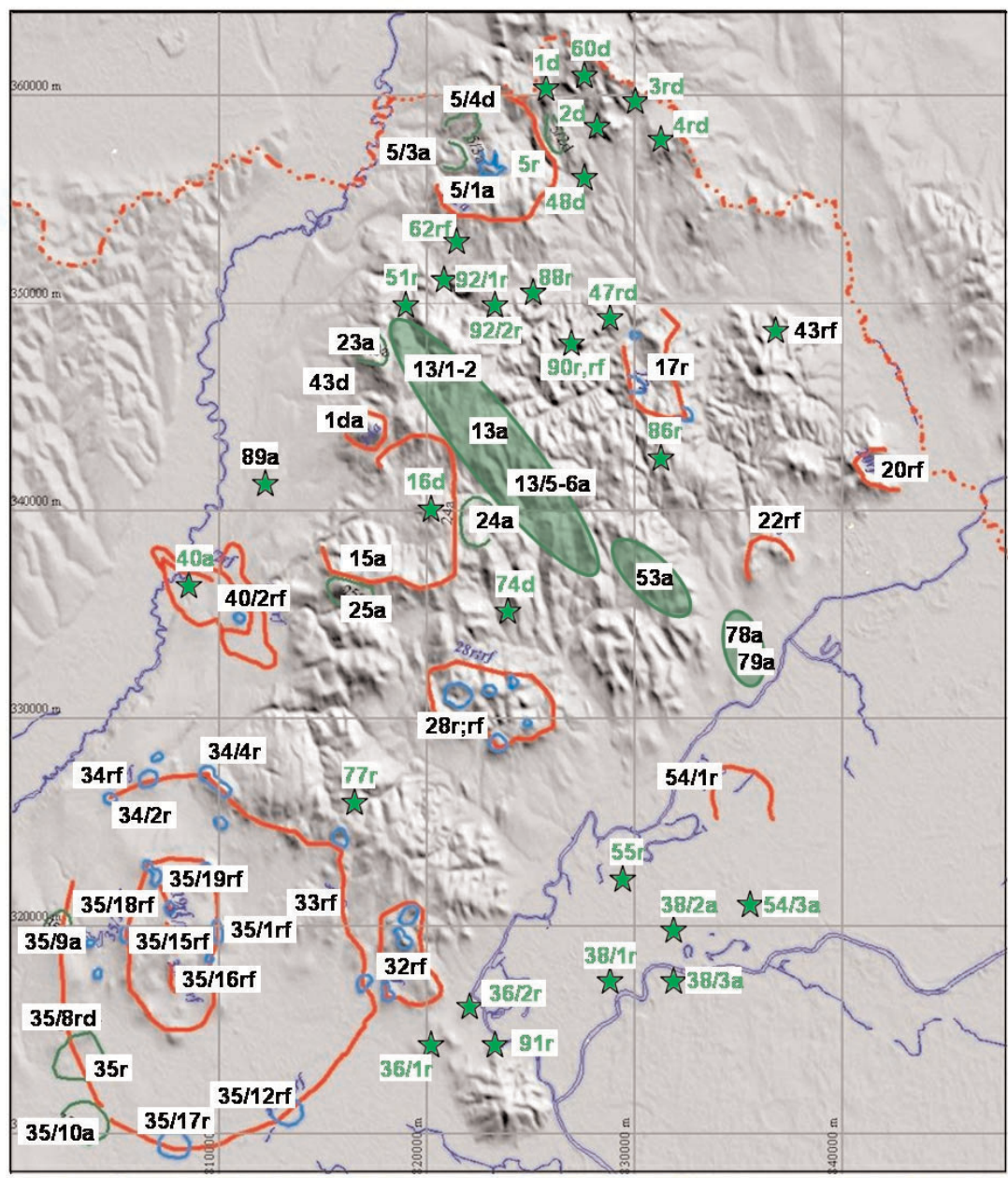

Fig. 11

Caldera structures (red lines), single lava domes (green stars), volcanic fissures (green poligons) (T. Zelenka, J. Kiss) 
The text and the attached tables include the rock name and the exploration methods indicating the given volcanic center (geology, remote sensing, gravity, magnetics, radiometry and geochemistry). Generally there were the congruent results of more than one method applied to indicate a supposed center. In the area covered with young sediments (Bodrogköz, Szerencs Creek, Hernád Valley) the buried eruption centers and the type of the lava and pyroclastic bodies in depth were mostly only identified by geophysical data, as outcrops and

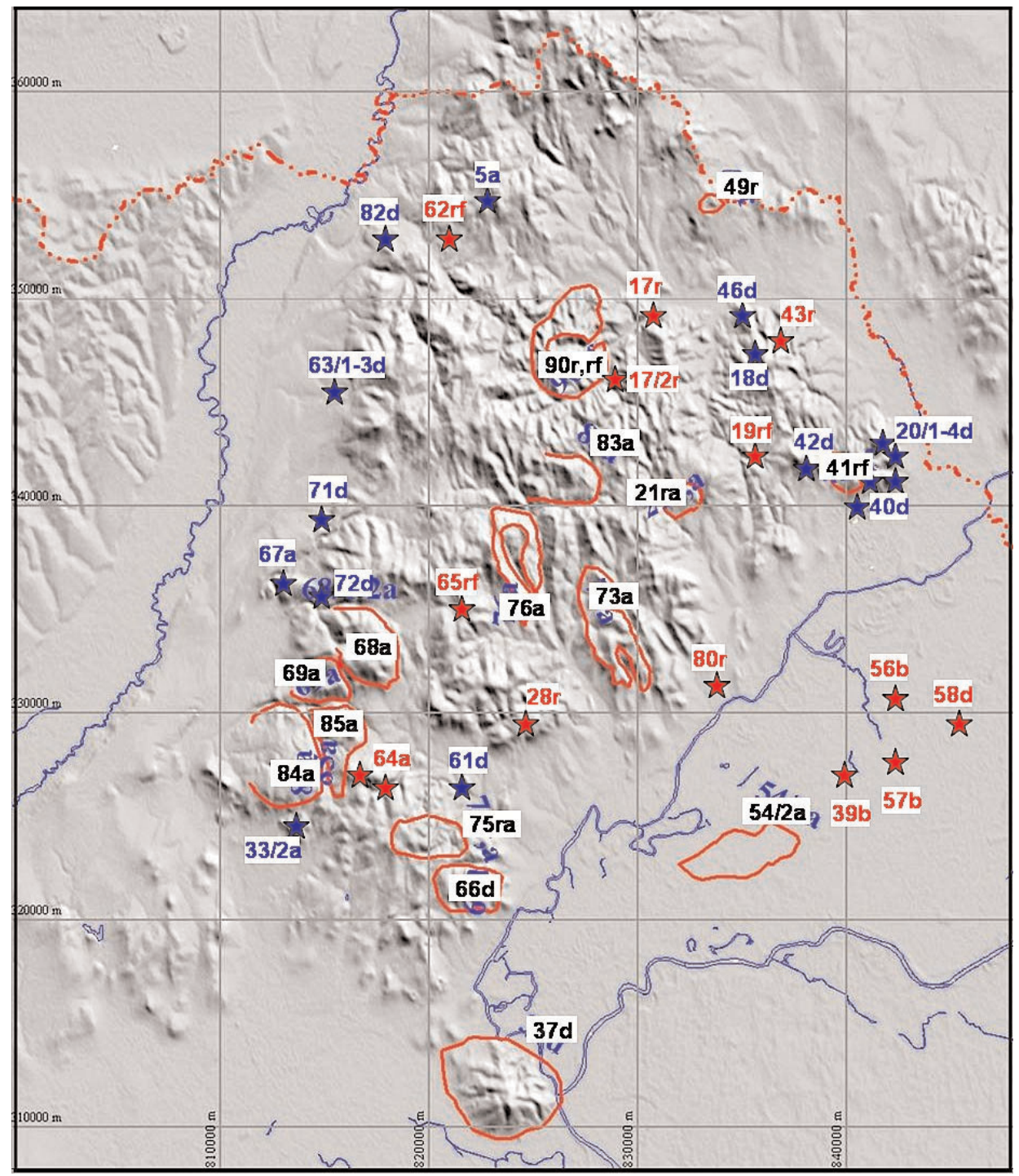

Fig. 12

Subvolcanic bodies (blue stars), diatremes (red stars), stratovolcanoes (red lines) (T. Zelenka, J. Kiss) 
boreholes were scarce or lacking. Beyond the 90 centers identified by complex geologic and remote sensing data in this study, we estimate their number to be nearly twice as many based on geophysical interpretation.

\section{Caldera structures}

These are oval ring structures with a diameter of several $\mathrm{km}$. Typical features are the escarpments with sharp morphology and the diversified inner parts. Collapsed calderas show a considerable difference of level between the escarpments and the inner part. The escarpments consist typically of multiple bedded, hard pyroclastics, with a series of acidic (rhyolite, dacite) lava domes and intermediate (andesite, andesitic dacite) parasitic cones on the top. In some cases subsequent subvolcanic bodies can be recognized beside the escarpment. More than $100 \mathrm{~m}$-thick pyroclastic flows, ignimbrites, hyaloclastic and peperitic lavas which flowed into water and tuffites with fauna are characteristic of the inner parts of the calderas. In the central zone subsequently intruded lava domes rise above these. Hard silicified 'cap rocks' originating from the hydrothermal water basins of the postvolcanic activity are widely distributed here. In some cases these hydrothermal zones are situated between some rising domes. The zones of different structure and material are easy to distinguish on airborne geophysical maps, satellite imagery and SAR imagery (Fig. 11).

In the following section a list of the caldera structures identified so far is given. Some structures are also introduced in detail.

\section{Szerencs Caldera (34rf, 34/2r, 35rf, 35r and 35d in Fig. 11)}

The Szerencs Hills ("Inselbergs") and the Szerencs Creek Valley are regarded as part of a large, recently strongly eroded, partly tectonically subsided caldera, which had never previously been identified. The methods for identification of rhyolitic calderas are demonstrated in detail with this example.

The satellite scene, the total magnetic gradient map (Fig. 6) and the Celebration-2000 CEL-4 deep seismic section and the velocity distribution maps (Hegedôs et al. 2002) outline a buried ring structure of approximately $25 \mathrm{~km}$ diameter between Abaújszántó and Szerencs. The radar, filtered gravimetry and magnetic total gradient maps also outline the inner and edge zones of the caldera as ring-shaped steps. In the center there is a gravity source body of 1-2 km in diameter at 900-1700 m depth; it is possibly a buried subvolcanic body. On the surface it is surrounded by small, composite rhyolite-dacite domes and their lava flows of 1-2 $\mathrm{km}$ in diameter along a circle 3-6 km in diameter at Monok (35/4-5rf, 15-19rf), Golop (35/3r) and Ond (35/1rf), belonging to the inner caldera structure. These are shown as filtered gravimetric and radiometric maxima. Their measured $\mathrm{K} /$ Ar age is 11.3-12.2 \pm 0.5 Ma (Molnár and Pécskay 2002). 
Table 1

Summary of the eruption centers

\begin{tabular}{|c|c|c|c|c|c|c|c|c|c|c|}
\hline & \multirow[t]{2}{*}{ Name of the volcanic centre } & \multicolumn{2}{|c|}{$\begin{array}{c}\text { Coordinates of the } \\
\text { center }\end{array}$} & Size & \multirow[b]{2}{*}{$a$} & \multirow[b]{2}{*}{$\mathrm{b}$} & & & & \\
\hline & & $\mathrm{x}$ & $\mathrm{Y}$ & $\mathrm{m} \times \mathrm{m}$ & & & $\mathrm{c}$ & d & $\mathrm{e}$ & $f$ \\
\hline & Szerencs Caldera & & & & & & & & & \\
\hline $34 / 1 \mathrm{rf}$ & Abaújszántó, Fehér Hill, rhyolite tuff (ignimbrite) & 807079 & 828216 & $1500 \times 1000$ & + & & + & + & + & + \\
\hline $34 / 2 \mathrm{r}$ & Abaújszántó, Sulyom Hill, rhyolite dome & 806438 & 326949 & $750 \times 750$ & + & & & & + & + \\
\hline $34 / 3 r$ & Abaújszántó, Süveges Hill, rhyolite dome & 804745 & 326183 & $800 \times 400$ & + & & + & & & \\
\hline $34 / 4 \mathrm{r}$ & Abaújszántó, Sátor - Krakó Hill, rhyolite dome & 809437 & 327340 & $2000 \times 1000$ & + & + & & & + & \\
\hline $35 / 1$ rf & Ond, Kassa Hill, rhyolite tuff (ignimbrite) & 807950 & 318146 & $1500 \times 1000$ & + & & + & & + & + \\
\hline $35 / 2 \mathrm{rf}$ & Szerencs, Fekete Hill, rhyolite tuff flow & 807600 & 317400 & $500 \times 500$ & + & + & & & + & \\
\hline $35 / 3 \mathrm{r}$ & Golop, Somos, rhyolite dome & 809357 & 322883 & $1500 \times 1000$ & + & + & + & & + & + \\
\hline $35 / 4 r, r f$ & Monok, Ör Hill - Szölö Hill, rhyolite dome with ignimbrite & 806498 & 322893 & $1500 \times 800$ & + & & & & + & + \\
\hline $35 / 5 r$ & Monok, Szentes Hill, rhyolite dome & 807690 & 320960 & $1000 \times 500$ & + & + & & & + & + \\
\hline $35 / 6 \mathrm{r}$ & Monok, Ing-vár, rhyolite dome & 803804 & 319177 & $250 \times 250$ & + & +1 & & & & + \\
\hline $35 / 7 \mathrm{r}$ & Monok, Kaptár - Pipiske, rhyolite dome & 803124 & 317750 & $1000 \times 1500$ & + & & & & + & \\
\hline $35 / 8 \mathrm{rd}$ & Legyesbénye, Majos Hill, rhyodacite dome & 803363 & 215076 & $500 \times 750$ & + & & + & + & + & + \\
\hline $35 / 9 a$ & Megyaszó, Nagy-Répás, andesite dome & 802602 & 319868 & $1500 \times 750$ & + & + & + & + & & \\
\hline $35 / 10 a$ & Legyesbénye, Dobogó, andesite & 803500 & 310500 & $500 \times 750$ & + & & + & + & & \\
\hline $35 / 11 \mathrm{r}$ & Taktaszada, rhyolite dome (buried) & 808000 & 309550 & $1500 \times 1500$ & & & + & + & & \\
\hline $35 / 12 r$ & Prügy, rhyolite dome (buried) & 813000 & 311500 & $1500 \times 1500$ & & & + & + & + & \\
\hline $35 / 13 d$ & Monok, Zsebrik, dacite dome & 805436 & 319698 & $1500 \times 600$ & + & & + & & + & \\
\hline $35 / 14 \mathrm{r}$ & Tállya, Patócs Hill - Akasztó Hill, rhyolite dome & 805486 & 319698 & $1500 \times 600$ & + & & + & & + & + \\
\hline $35 / 15 \mathrm{rf}$ & Monok, Kövágótetö, rhyolite tuff inside the caldera & 807740 & 319370 & $1500 \times 1000$ & + & + & & & & \\
\hline $35 / 16 \mathrm{rf}$ & Monok, Nyires, rhyolite tuff inside the caldera & 807680 & 317520 & $2000 \times 1500$ & + & + & & & & \\
\hline $35 / 19 \mathrm{rf}$ & Monok, Szentes Hill, rhyolite tuff inside the caldera & 807370 & 331350 & $1500 \times 1000$ & + & + & & & + & + \\
\hline $33 / 1 r, r f$ & Tállya, Dorgó-tetö - Fürdös-tetö, rhyolite, ignimbrite & 815958 & 323955 & $2500 \times 2000$ & + & & + & & + & \\
\hline & Hollóháza Caldera & & & & & & & & & \\
\hline $5 a, r$ & Hollóháza - Telkibánya, andesite with rhyolite dome & 821506 & 357136 & $7000 \times 8000$ & + & + & + & + & + & + \\
\hline $5 r$ & Hollóháza, Pál Hill, rhyolite dome & 822750 & 356450 & $1000 \times 1000$ & + & + & + & & + & \\
\hline $5 / 2 d$ & Hollóháza, Május Hill, dacite dome with lava flow & 826020 & 357880 & $1000 \times 250$ & + & + & & & & \\
\hline $5 / 3 a$ & Pányok, Tilalmas, andesite parasitic cone & 821510 & 357140 & $1000 \times 1000$ & + & + & + & + & & \\
\hline $5 / 4 d$ & Kéked, Les Hill, dacite parasitic cone & 821570 & 358590 & $1000 \times 1000$ & + & + & & + & & \\
\hline $5 / 5 \mathrm{a}$ & Telkibánya, Gyepü Hill, andesite subvolcano & 823080 & 354690 & $1500 \times 1500$ & + & + & & & + & + \\
\hline & Regéc caldera & & & & & & & & & \\
\hline $15 a$ & Regéc, andesite caldera & 818792 & 339965 & $8000 \times 7000$ & + & + & & & & \\
\hline $16 \mathrm{~d}$ & Regéc, Castle Hill, dacite (dome) & 820184 & 339844 & $500 \times 500$ & + & + & + & + & + & + \\
\hline $14 a$ & Regéc, Gergely Hill, andesite & 817480 & 343881 & $500 \times 500$ & + & + & & + & & \\
\hline $25 a$ & Arka, Magoska, andesite & 816118 & 336239 & $2000 \times 2000$ & + & + & + & + & & \\
\hline $24 a$ & Óhuta, Zabarla, andesite & 802602 & 319868 & $1000 \times 500$ & + & + & & & & \\
\hline $23 a$ & Gönc, Borsó Hill, andesite & 817370 & 347306 & $1000 \times 1000$ & + & + & & + & & \\
\hline & Boldogkö caldera & & & & & & & & & \\
\hline $40 / 1 \mathrm{r}, \mathrm{rf}$ & Boldogkőváralja, Szentiván Hill, rhyolite caldera & 810750 & 335000 & $5700 \times 1700$ & + & + & & + & + & \\
\hline $40 / 2$ rf & Boldogkőváralja, Boldogkő Castle, rhyolite tuff dam & 811600 & 335500 & $1500 \times 250$ & + & + & + & + & + & \\
\hline $40 / 4$ rf & Vizsoly, quarry, degassed rhyolite tuff, ignimbrite flow & 810500 & 339000 & $1000 \times 1000$ & + & + & & & & \\
\hline $40 / 3$ a & Alsócéce, andesite dome (buried) & 808610 & 336090 & $800 \times 800$ & & & + & + & & \\
\hline & Viss caldera & & & & & & & & & \\
\hline $54 / 1 \mathrm{rf}$ & Viss, Patkó-zug, rhyolite tuff (buried) & 835000 & 326600 & $2500 \times 2500$ & & & + & + & & \\
\hline $54 / 2$ a & Viss, Patkó-zug, andesite dome (buried) & 835020 & 323030 & $500 \times 500 ?$ & & & + & + & & \\
\hline & Pálháza caldera & & & & & & & & & \\
\hline 17 & Pálháza, Som Hill, rhyolite dome and lava flow & 831181 & 348022 & $5000 \times 2000$ & + & + & & & + & + \\
\hline $17 / 1 \mathrm{r}$ & Pálháza, Gyöngykö Hill, rhyolitic perlite dome, diatreme & 830300 & 349200 & $1000 \times 1000$ & + & + & & & + & \\
\hline $17 / 2 r$ & Kishuta, Laczkó Hill, rhyolitic perlite dome & 829200 & 346800 & $600 \times 400$ & + & + & & & + & \\
\hline $47 \mathrm{rd}$ & Nagybózsva, Páska-tető, rhyodacite dome, diatreme & 828868 & 348864 & $600 \times 500$ & + & + & & & + & \\
\hline $17 / 3 \mathrm{r}$ & Nagyhuta, Gilevár, rhyolitic perlite dome & 828200 & 346400 & $500 \times 500$ & + & + & & & + & \\
\hline 22 & Sárospatak, Király Hill, Megyer Hill & 836379 & 338327 & $3000 \times 1000$ & + & + & & & + & \\
\hline & Erdőhorváti - Erdőbénye caldera & & & & & & & & & \\
\hline $28 \mathrm{r}, \mathrm{rf}$ & $\begin{array}{l}\text { Erdöbénye - Tolcsva - Erdöhorváti, rhyolite (with ignimbrite } \\
\text { flows) }\end{array}$ & 823019 & 331471 & $5000 \times 3000$ & + & + & + & + & + & \\
\hline $28 / 6 r f$ & Ránytető, rhyolite ash flow & 824721 & 329353 & $750 \times 500$ & + & + & + & + & + & \\
\hline $28 / 1 \mathrm{r}$ & Erdöhorváti, Szokolya, rhyolite dome & 823019 & 331471 & $750 \times 750$ & + & + & & & + & \\
\hline $28 / 2 r$ & Nagy-Páca, rhyolite dome & 824831 & 330755 & $500 \times 500$ & + & + & & & + & \\
\hline $28 / 3 r$ & Rakottyás, rhyolite dome & 824851 & 329779 & $500 \times 500$ & + & + & & & + & \\
\hline $28 / 4 \mathrm{r}$ & Peres Hill, rhyolite dome & 823479 & 328852 & $750 \times 750$ & + & + & & & + & \\
\hline $28 / 5 \mathrm{r}$ & Nagy-Mondoha, rhyolite dome & 821086 & 330825 & $1250 \times 750$ & + & + & & & + & \\
\hline & Mád caldera & & & & & & & & & \\
\hline 32 & Mád, Diósd, andesite and rhyolite (subvolcanic and dome) & 818600 & 320500 & $2000 \times 2000$ & + & + & & & + & + \\
\hline $32 / 1 \mathrm{r}, \mathrm{ff}$ & Mád, Király Hill, rhyolite dome, rhyolite tuff, ignimbrite & 818950 & 318210 & $1000 \times 500$ & + & + & & & + & \\
\hline $32 / 2$ rf & Mád, Bomboly, rhyolite tuff, ignimbrite & 818170 & 320330 & $500 \times 500$ & + & + & & & + & + \\
\hline $32 ! 3 r, r f$ & f Mád, Szemere-tetö, rhyolite, fallen rhyolite tuff & 818600 & 314800 & $500 \times 500$ & + & + & & & + & \\
\hline $32 / 4 \mathrm{r}, \mathrm{rf}$ & f Bodrogkeresztúr, Kakas, rhyolite, rhyolite tuff & 820400 & 317400 & $1000 \times 500$ & + & + & & & + & \\
\hline & Volcanic fissures & & & & & & & & & \\
\hline $13 / 2 / 1 \mathrm{a}$ & Telkibánya, Magas-Tér - Hemzsö-bérc, andesite & 820154 & 349580 & $3000 \times 500$ & + & + & & + & & \\
\hline $13 / 2 / 2 a$ & Telkibánya, Holló-kö - Nagy-Sertés Hill, andesite & 822388 & 344982 & $2000 \times 500$ & + & + & & + & & \\
\hline $13 / 1 / 1 \mathrm{a}$ & Gönc, Amádé-vár - Téglás-kö, andesite (3 samples) & 818832 & 347847 & $1500 \times 1000$ & + & + & & + & & \\
\hline $13 / 1 / 2 a$ & Hejce, Bán Hill - Szár-kö, andesite & 819713 & 344261 & $1000 \times 1000$ & + & + & & + & & \\
\hline $13 / 6 a$ & Nagyoldal-tetö - Nagy-Péter-mennykö, andesite & 822830 & 342200 & $2000 \times 700$ & + & + & & + & & \\
\hline $13 / 5 a$ & llonka Meadow - Sólyom-bérc, andesite & 820370 & 344810 & $1000 \times 700$ & + & + & & + & & \\
\hline $13 / 2 / 3 a$ & Regéc, Nagy-Bekecs - Tokár-tetö, andesite & 821707 & 347607 & $2500 \times 700$ & + & + & & + & & \\
\hline \begin{tabular}{|l|l}
$53 a$ \\
\end{tabular} & Komlóska, Nagy-Papaj, andesite & 830871 & 336214 & $1000 \times 1000$ & + & + & + & + & & \\
\hline
\end{tabular}

a - Geology, b - Remote sensing, c- Gravity, d-Magnetics, e - Radiometry, f - Geochemistry 
Table 1

(Cont.)

\begin{tabular}{|c|c|c|c|c|c|c|c|c|c|c|}
\hline & \multirow[t]{2}{*}{ Name of the volcanic centre } & \multicolumn{2}{|c|}{$\begin{array}{l}\text { Coordinates of the } \\
\text { center }\end{array}$} & Size & \multirow[b]{2}{*}{$\mathrm{a}$} & \multirow[b]{2}{*}{ b } & \multirow[b]{2}{*}{ c } & \multirow[b]{2}{*}{ d } & \multirow[b]{2}{*}{$\mathrm{e}$} & \multirow[b]{2}{*}{ 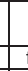 } \\
\hline & & $\mathrm{X}$ & $\mathrm{Y}$ & $\mathrm{m} \times \mathrm{m}$ & & & & & & \\
\hline $78 / 1-3 a$ & $\begin{array}{l}\text { Sárospatak, Szent Vince Hill - Páncél Hill, andesite - } \\
\text { Hercegkút, Gombos }\end{array}$ & 834717 & 334671 & $3000 \times 200$ & + & + & + & + & & \\
\hline $79 / 1-2 a$ & Sárospatak, Mandulás - Kutya Hill - Bodroghalász andesite & 835198 & 334176 & $3000 \times 200$ & + & + & + & + & & \\
\hline & Subvolcanic bodies, intrusions & & & & & & & & & \\
\hline $46 \mathrm{~d}$ & Kovácsvágás, Baradla, dacite subvolcano & 831141 & 342514 & $1000 \times 200$ & + & + & & + & + & \\
\hline $20 / 1-3 d$ & $\begin{array}{l}\text { Sátoraljaújhegy, Magas Hill, Szár Hill, Vár Hill, Sátor Hill, } \\
\text { dacite subvolcanoes }\end{array}$ & 841948 & 342243 & $3000 \times 3000$ & + & + & + & + & & \\
\hline $20 / 4 d$ & Sátoraljaújhely, Vár Hill, dacite subvolcano & 841397 & 341372 & $250 \times 250$ & & & & & & \\
\hline $40 \mathrm{~d}$ & Sátoraljaújhely, Néma Hill, dacite subvolcano & 840836 & 339885 & $2000 \times 1000$ & + & + & & + & & \\
\hline $42 \mathrm{~d}$ & Rudabányácska, Száva Hill, dacite subvolcano & 838252 & 342043 & $2000 \times 1000$ & + & + & + & + & + & + \\
\hline $18 d$ & $\begin{array}{l}\text { Kovácsvágás - Vágáshuta, Fekete Hill - Osztra Hill NE, } \\
\text { dacite subvolcano }\end{array}$ & 836109 & 346911 & $1500 \times 600$ & + & + & + & + & & \\
\hline $17 / 2 r$ & \begin{tabular}{|l|l|l|} 
Pálháza- Kishuta, Gilevár, perlite extrusion \\
\end{tabular} & 831181 & 348022 & $500 \times 500$ & + & + & + & & + & \\
\hline $33 / 2 a$ & $\begin{array}{l}\begin{array}{l}\text { Tállya, Gomboska - Kopasz Hill, pyroxene andesite } \\
\text { subvolcano }\end{array} \\
\end{array}$ & 813704 & 324536 & $800 \times 800$ & + & + & & + & & \\
\hline $61 \mathrm{~d}$ & Erdőbénye, Mulató Hill, pyroxene andesite subvolcano & 821677 & 326143 & $2000 \times 500$ & + & + & + & + & & \\
\hline $63 \mathrm{~d}$ & Hejce, Tilalmas, 3 samples of $\mathrm{N}-\mathrm{S}$ dacite subvolcanoes & 815236 & 345593 & $2000 \times 1000$ & + & + & + & + & + & \\
\hline $67 a$ & Boldogkőváralja, Tó Hill, andesite & 812803 & 336439 & $2000 \times 1000$ & + & + & + & + & & \\
\hline $71 \mathrm{~d}$ & Korlát, Répás, dacite & 814736 & 339594 & $250 \times 250$ & + & + & & + & + & \\
\hline $82 \mathrm{~d}$ & Gönc, Vas Hill, dacite & 817650 & 353055 & $1000 \times 1000$ & + & + & + & + & + & + \\
\hline & Diatremes & & & & & & & & & \\
\hline $64 a$ & Erdöbénye, Szokolya, andesite & 817360 & 326894 & $500 \times 500$ & + & + & & + & & \\
\hline $65 a$ & Baskó, Horváthkút Meadow, rhyolite tuff & 821596 & 334842 & $500 \times 500$ & + & 63 & + & & & \\
\hline $62 \mathrm{rf}$ & Telkibánya, Youth camp, rhyolite tuff & 820595 & 352695 & $1500 \times 1000$ & + & + & & & + & \\
\hline $19 \mathrm{rf}$ & Sárospatak, Nyilazólyuk (Nyilkút), rhyolite tuff & 835889 & 341983 & $500 \times 500$ & + & & + & & & \\
\hline $39 b$ & Sárospatak, Apróhomok, basalt & 840000 & 327000 & $500 \times 500$ & + & & & + & & \\
\hline $28 / 6 \mathrm{rf}$ & Erdőbénye, Kőgát, rhyolite tuff & 824721 & 329353 & $250 \times 250$ & + & + & & & + & \\
\hline $43 \mathrm{rf}$ & Mikóháza, Baradla, rhyolite tuff & 837051 & 347962 & $500 \times 500$ & + & + & & & + & + \\
\hline $56 \mathrm{~b}$ & Vajdácska, Várhomok, basalt? (buried) & 842410 & 330650 & $500 \times 500 ?$ & & & + & + & & \\
\hline $57 \mathrm{~b}$ & Dorkó, Cseredülő, basalt? (buried) & 842250 & 327810 & $500 \times 500 ?$ & & & + & + & & \\
\hline $58 \mathrm{~d}$ & Bodroghalom, Nyirtanya, dacite (buried) & 845630 & 329520 & $500 \times 500 ?$ & & & + & + & & \\
\hline $80 \mathrm{ba}$ & Sárazsadány, Mancsalka, andesite & 833680 & 331370 & $1000 \times 1000$ & & & + & + & & \\
\hline & Stratovolcanoes & & & & & & & & & \\
\hline $37 d$ & Tokaj, Nagy Hill, dacite stratovolcano & 823925 & 311375 & $6000 \times 6000$ & + & + & & + & & \\
\hline $66 \mathrm{~d}$ & Szegi, Cigány Hill, dacite stratovolcano & 821570 & 321780 & $3500 \times 2000$ & + & + & & + & & \\
\hline $41 \mathrm{rf}$ & Sátoraljaújhely, Fekete Hill - Rudabányácska, Bánya Hill & 840406 & 341121 & $2000 \times 1000$ & + & & & & + & + \\
\hline $73 a$ & Tolcsva, Fekete Hill, andesite stratovolcano & 828527 & 333670 & $6500 \times 2000$ & + & + & & & & \\
\hline $21 \mathrm{~d}, \mathrm{a}, \mathrm{r}$ & Makkoshotyka, Katuska Hill, dacite - andesite - rhyolite & 831993 & 340621 & $2000 \times 2000$ & + & + & + & + & + & + \\
\hline $75 a, r f, r$ & Erdöbényefürdő, Hollóstetö, andesite - rhyolite tuff, rhyolite & 819653 & 323684 & $3500 \times 2100$ & + & & & + & + & + \\
\hline $76 a$ & Óhuta, Magas Hill, andesite & 823910 & 338497 & $4000 \times 2000$ & + & + & & + & & \\
\hline $83 a$ & Háromhuta, Nagy Körös Hill - Nagykirályos, andesite & 826660 & 341790 & $3500 \times 2200$ & + & + & + & + & & \\
\hline $84 a$ & Abaújszántó, Molyvás, andesite & 813500 & 328000 & $5000 \times 3000$ & + & + & + & + & & \\
\hline $85 a$ & Abaújkér, Bánya Hill, andesite & 815680 & 328730 & $4500 \times 2000$ & + & + & & + & & \\
\hline $68 a$ & Sima, Nagykorsós-Somberek, andesite & 816789 & 332528 & $3000 \times 3500$ & + & + & + & + & & \\
\hline $54 / 2 a$ & Viss, Fazekas-zug, andesite (buried) & 835020 & 323030 & $6000 \times 2500$ & & & + & + & & \\
\hline $90 \mathrm{rf}, \mathrm{r}$ & $\begin{array}{l}\text { Nagybózsva, Fekete Hill - Csattantyú Hill, rhyolite tuff, } \\
\text { rhyolite }\end{array}$ & 827000 & 347500 & $5000 \times 5000$ & + & + & + & & + & \\
\hline & Single lava domes & & & & & & & & & \\
\hline $51 \mathrm{r}$ & Telkibánya, Varga Hill - Nagy Valley, rhyolite dome & 819400 & 349990 & $500 \times 500$ & + & & + & + & & \\
\hline $46 r$ & Nagyhuta, Jakabvár Hill, Nagyfulón, rhyolite domes & 835238 & 348934 & $500 \times 500$ & & & + & + & & + \\
\hline 3 rd & Pusztafalu,Tolvaj Hill, rhyodacite dome & 830110 & 360031 & $1000 \times 1000$ & + & & + & & & + \\
\hline 4 rd & Füzérkajata, Hársas Hill, rhyodacite dome & 831832 & 357587 & $800 \times 800$ & + & & + & & & \\
\hline $48 a$ & Füzérkomlós, Akasztó Hill, andesite dome & 827776 & 355564 & $\begin{array}{c}500 \times 500 \\
2000 \times 2000\end{array}$ & + & & & & & \\
\hline $1 d$ & Hollóháza, Vágott Hill, dacite dome & 825690 & 360650 & $1000 \times 500$ & + & + & + & & & \\
\hline $2 \mathrm{~d}$ & Füzér Castle, dacite neck & 828144 & 358246 & $500 \times 500$ & + & + & + & & & + \\
\hline $49 r$ & Füzérradvány, Korom Hill, rhyolite dome & 833645 & 354557 & $\begin{array}{c}2000 \times 2000 \\
500 \times 500\end{array}$ & + & & + & & & + \\
\hline $60 \mathrm{~d}$ & Pusztafalu, Tolvaj Hill, dacite dome & 827520 & 360480 & $750 \times 750$ & + & + & + & & & \\
\hline $63 d$ & Hejce, Tilalmas, dacite dome & 815236 & 345593 & $500 \times 250$ & + & + & + & + & + & + \\
\hline $74 \mathrm{~d}$ & Erdöhorváti, Eperjeske, dacite dome & 823790 & 334970 & $700 \times 500$ & + & + & & & & \\
\hline $77 \mathrm{r}$ & Erdöbénye, Faragványos, rhyolite & 816660 & 326030 & $1200 \times 1500$ & + & + & & & + & + \\
\hline $47 \mathrm{rd}$ & Nagybózsva, Páska Hill, rhyolite dome & 828868 & 348864 & $500 \times 500$ & + & + & & & + & \\
\hline $91 \mathrm{r}$ & Tokaj, Lebuj Inn, rhyolite dome & 823400 & 314200 & $250 \times 250$ & + & & & & + & \\
\hline $92 / 1 \mathrm{r}$ & Telkibánya, Cser Hill, rhyolite-perlite dome & 821600 & 350600 & $1200 \times 400$ & + & + & & & + & \\
\hline $92 / 2 \mathrm{r}$ & Telkibánya, Ork-Ógönc, rhyolite-perlite domes & 823600 & 349400 & $1500 \times 700$ & + & + & & & + & \\
\hline $36 / 2 r$ & Bodrogkeresztúr, Dereszla, rhyolite dome & 822110 & 316240 & $500 \times 500$ & + & + & & & + & \\
\hline $36 / 1 \mathrm{r}$ & Tarcal, Terézia Hill, rhyolite dome & 820150 & 324140 & $500 \times 500$ & + & + & + & & + & \\
\hline $38 / 1 \mathrm{r}$ & Zalkod, Lukató, rhyolite? dome (buried) & 828710 & 317540 & $?$ & & + & + & & & \\
\hline $38 / 2 a$ & Zalkod, Jakab Hill, andesite (buried) & 831770 & 319930 & $?$ & & & + & + & & \\
\hline $38 / 3 a$ & Szabolcs, Földvár (motte), andesite lava dome (drillhole) & 831710 & 317220 & $500 \times 500 ?$ & + & & + & + & & \\
\hline $54 / 3 a$ & Viss, Fazekas-zug, andesite lava dome (buried) & 835660 & 320900 & $500 \times 500 ?$ & & & + & + & & \\
\hline $54 / 1 \mathrm{r}$ & Viss, Patkó-zug, rhyolite dome (buried) & 834430 & 326470 & $500 \times 500 ?$ & & & + & & + & \\
\hline $55 \mathrm{r}$ & Szegi, Kásod Meadow, rhyolite dome (buried) & 829920 & 321980 & $500 \times 500 ?$ & & + & + & & & \\
\hline
\end{tabular}

a - Geology, b-Remote sensing, c - Gravity, d-Magnetics, e - Radiometry, f - Geochemistry 
According to the geologic mapping (Gyarmati and Zelenka 1968a and b) and structural and mineral resource exploration boreholes, there is ignimbrite as well as fallen and redeposited tuff of 200-350 m thickness inside the caldera, between the lava domes. These indicate five horizons of eruptions (Zelenka 1964).

Andesite domes (Megyaszó 35/9a, Legyesbénye 35/10a) and subvolcanoes (Tállya 33/2a) showing local magnetic and gravity maxima are uplifted at the edges of the outer circle of the large caldera. Rhyolite volcanoes (Monok 35/6-7r, Abaújszántó 34/1-4r,rf, Tállya 33/1rf, Mád 33/3r, rf, Prügy 35/17r) can be outlined with filtered gravimetric and radiometric total intensity maxima. The determined $\mathrm{K} /$ Ar age of these volcanic formations is $11.6 \pm 0.4 \mathrm{Ma}$ (Molnár and Pécskay 2002).

In the thermal water-charged geyser basins of the postvolcanic centers limnoquartzite, bentonite and diatomite were deposited (Rátka: Koldu, Kerektölgyes, Hercegköves, Új Hill (33/5u); Szerencs: Aranka-tető (35/20u); Bekecs: Kis Hill (35/21u); Monok: Zsebrik). In these basins As and $\mathrm{Hg}$ were enriched. Low sulfidization (LS) type, Au-bearing quartz veins (ore indications) can be found on Fuló Hill at Legyesbénye (35/22u) and on Bomboly at Mád $(32 / 2 u)$ in areas subjected to potassic metasomatism. Postvolcanic activity was accompanied by kaolinitization and alunitization. The K/Ar age of the latter is 10.4-12.1 \pm 0.7 Ma (Molnár and Pécskay 2002). Diatomite deposits were formed in fallen, bedded rhyolite tuff deposited in water (Zelenka 1967).

The bulk of ignimbritic ash-flow tuffs was also deposited in water. The glassy material of the tuffs is strongly zeolitized. At Rátka the welded pumiceous tuff contains spherical bombs of $20-40 \mathrm{~cm}$ diameter, formed from the same material, originating from the ignimbritic flow of the Fürdős-tető eruption center. At Abaújszántó and Bánya Hill submarine exhalative $\mathrm{Pb}-\mathrm{Zn}-\mathrm{Sb}$ ore lenses were formed in the rhyolite tuff or tuffite and in the accompanying Sarmatian sediments (Pentelényi 1967).

Boldogkóvár Caldera (40rf, 40a in Fig. 11)

Viss Caldera (54a, $54 r$ in Fig. 11)

Hollóháza Caldera (5r, $5 a$ in Fig. 11)

A double ring of a caldera of $7-8 \mathrm{~km}$ in diameter was recognized on a satellite image between Hollóháza and Telkibánya (Fig. 11). At the edge of the caldera a series of andesitic and dacitic parasitic volcanic cones can be found (Liffa 1953). On the northern part of the caldera there are rhyolite domes and their lava flows with large K, Th and U anomalies, which broke through Sarmatian rhyolite tuff and argillaceous sediments (Kiss and Zelenka 2009). On the southern part, at the gravity maximum of Telkibánya Kánya Hill and Gyepü Hill (5/3a, 5/7a) the $2.5 \times 2.0 \mathrm{~km}$-sized caldera structure, open in $\mathrm{V}$-shape to the $\mathrm{S}$, can be traced down to a depth of 1500-1600 m, accompanied by K, As, Au, Ag and Sb enrichment on the surface with 20 nearly N-S and NNE-SSW-striking, LS-type epithermal precious metal ore veins $(5 / 7 \mathrm{u})$. The K/Ar age is 11.8-13.1 Ma (Pécskay et al. 1986; Molnár and Pécskay 2002). 
The Telkibánya-2 structural borehole found rhyolitic and dacitic tuff complex over the Badenian clay marl (Székyné Fux 1970). The original stratovolcanic amphibole andesite was propylitized and intruded by the K-metasomatic subvolcanic andesite on the caldera edge (Molnár and Zelenka 1995).

Regéc Caldera (15a, 15d in Fig. 11)

Pálháza Caldera (17r in Fig. 11)

Erdóbénye Caldera (28r in Fig. 11)

Mád Caldera (30a, 30r, 32rf in Fig. 11)

Sárospatak Caldera (22rf in Fig. 11)

Sátoraljaújhely Caldera (20rf in Fig. 11)

\section{Volcanic fissures}

The hard, standing dykes form elevated crests and ridges on the surface. Near to the upstreaming zone the rock is steeply banded, while around the channels the pyroxene andesite lava beds dip $10-30^{\circ}$. The following occurrences were grouped here:

Telkibánya, Magastér - Resztelt-bérc - Hemzsó-bérc (13-3a in Fig. 11)

Telkibánya, Hollókố - Nagy-Sertés Hill (13-2a in Fig. 11)

Gönc, Amádévár - Téglás-kó - Bán Hill (13a in Fig. 11)

A nearly NNW-SSE striking pair of bands of gravity maximum and minimum in the direction Gönc, Amádévár - Téglás-kô - Bán Hill indicates a nearly 10 kmlong tectonic lineament (Fig. 11). This is also outlined on the satellite image along the Kis Creek. Three spots of magnetic $\Delta \mathrm{T}$ anomalies and total gradient maxima can be found at Amádévár, Téglás-kô and Bán Hill, each 1-1.5 km in diameter (Fig. 6). The lava beds show $60^{\circ}$ dip around the eruption centers and $10-35^{\circ}$ in the distal region. The ring structure at Téglás-kó on the satellite scene indicates a small-sized eruption centre. The K/Ar age of the lava cover (pyroxene andesite) at Sólyom Rock, belonging to this zone, is $11.9 \pm 0.52 \mathrm{Ma}$ (Pécskay in Zelenka et al. 2007).

Regéc, Kis-Bekecs - Nagy-bekecs - Pengóo-kó - Tokár-tetó and Nagyoldal-tető - NagyPéter-mennykó (13-5, 6/a in Fig. 11)

Komlóska, Nagy-Papaj (53/a in Fig. 11)

Sárospatak, W and SW part (78a/79/a in Fig. 11)

The pyroxene andesite lava bodies of two parallel, N-S striking, several km long and some $10 \mathrm{~m}$ wide series of volcanic fissures appear along the Hotyka Creek Fault, W and SW of Sárospatak, as weak magnetic and gravity anomalies. The K/Ar age of Páncél Hill and Szent Vince Hill (78/1-3a) on the western side is $12.5 \pm 0.9 \mathrm{Ma}$, while the age of the bodies along Hercegkút Gombos, Sárospatak Mandulás, Sárospatak Kutya Hill (79/1-2a), Sárospatak Vár Hill on the eastern side is $11.63 \pm 0.45 \mathrm{Ma}$ (Pécskay in Zelenka et al. 2007). 
Subvolcanic bodies, intrusions

These bodies consist of microholocrystalline or porphyry rocks with sharp morphological boundaries and often with columnar joints. On the gravity map these are denser bodies than their surroundings, extending to depth. Their texture changes downward; according to mining exploration data it becomes coarsely crystalline.

Kovácsuágás, Baradla (46/d in Fig. 12)

Sátoraljaújhely (20/rf 20/d in Fig. 12)

At Sátoraljaújhely, on the western edge of the uplifted (outcropping) basement there are andesitic-dacitic subvolcanic bodies in a pincer-shaped caldera, intruded into and affecting the Badenian faunal clay marl by contact. The location of the subvolcanoes on the satellite image is along the edge of the rhyolite tuff and tuffite caldera, in the line of Magas Hill, Szár Hill, Vár Hill, Sátor Hill and Kecskehát, corresponding to an oval magnetic and gravity anomaly. The $\mathrm{K} / \mathrm{Ar}$ age $(11.9 \pm 0.5 \mathrm{Ma})$ and the paleomagnetic results (reverse polarization, minor $\mathrm{W}$ rotation) indicate Sarmatian age.

Sátoraljaújhely, Néma Hill (20/d in Fig. 12)

Rudabányácska, Száva Hill (42/d in Fig. 12)

Kovácsvágás (E), Fekete Hill - Osztra Hill (18/d in Fig. 12)

Pálháza - Kishuta (17/r in Fig. 12)

Tállya, Kopasz Hill (33/a in Fig. 12)

The andesite of the subvolcanic body at Tállya - Kopasz Hill was intruded into loose pumiceous rhyolite tuff. The intrusion is supposed to have proceeded in two phases, based on the two kinds of pyroxene andesite. The light grey andesite with vertical columnar joints and sporadic sulfide dissemination was formed first. The K/Ar age of this intrusion is $11.7 \pm 1.1 \mathrm{Ma}$ (Pécskay et al. 1986). Later this cooled andesite was again intruded by a black, olivine-rich pyroxene andesite with folded columnar joints, partly along earlier joints, partly on the edge contact. The latest K/Ar measurements show 9.6 Ma age for the olivine-rich andesite (Pécskay, pers. comm).

Hejce, Tilalmas (63/d in Fig. 12)

Boldogkốváralja, Tekeres Valley (72/d in Fig. 12)

Boldogkóváralja, Tó Hill (69/a in Fig. 12)

Telkibánya, Kánya Hill - Gyepü Hill (5/a in Fig. 12)

Erdóbénye, Mulató Hill (61/d in Fig. 12)

The olivine-bearing hypersthene-augite dacite laccolith of Barnamáj (Kulcsár and Bartha 1971) was intruded into micro- and macrofaunal argillaceous tuffite and pumiceous, unwelded rhyolite tuff, metamorphosing these by contact. The body shows weak gravity and strong magnetic anomaly, indicating no extension in depth. The K/Ar age is $11.3 \pm 0.5 \mathrm{Ma}$. 
Korlát (71/d in Fig. 12)

Gönc, Hársas Hill - Vas Hill (82d in Fig. 12)

The pyroxene-amphibole dacite subvolcanic body of Hársas Hill and Vas Hill was intruded into Sarmatian faunal clay and pumiceous rhyolite tuff, leading to contact metamorphism. The Telkibánya-VIII borehole crossed this subvolcanic body in more than $100 \mathrm{~m}$ length (Ilkeyné Perlaki 1967, 1978). The K/Ar age is 11.6 $\pm 0.7 \mathrm{Ma}$.

Bodroghalom (58/d in Fig. 12)

\section{Diatremes}

Diatremes originated mainly from deeply rooted, highly explosive phreatomagmatic eruptions. A circular or ring-shaped local gravity minimum indicates the center of a diatreme within the basement regional gravity maximum. Andesite and andesitic dacite cause a weak magnetic total gradient maximum (Fig. 6).

In those sites where the basement or older volcanic bodies are crosscut by a rhyolite tuff vent, circular gravity minima can be observed as well. Some $10 \mathrm{~cm}$ scale rhyolite blocks, pumice lapilli and xenoliths of basement origin (limestone, micaschist, gneiss) prove the proximity of the explosive center. The expelled debris of the rhyolite tuff is unsorted; its cement is in most cases pumiceous, fresh volcanic glass.

\section{S of Vágáshuta, Nyúl Spring (19/rf in Fig. 12)}

A circular gravity minimum of $1 \mathrm{~km}$ diameter was observed on the top a maximum at Nyúl Spring, S of Vágáshuta. Large $(20-40 \mathrm{~cm})$ xenoliths from basement rocks (carbonates and micaschist) are embedded in welded ignimbritic ash-flow tuff, indicating vent-proximal eruptive facies.

Quarries exploited the material of repeated lava breakthroughs younger than the surrounding explosion vents of pyroclastic flows and hyaloclastite breccia inside the calderae. For example, there is fresh pyroxene andesite in the Kmetasomatized andesite of the Kánya and Gyepü Hills at Telkibánya (5/7; Horváth and Zelenka 1997) and a perlite lava extrusion in the hyaloclastite breccia of the Gyöngykő Hill at Pálháza (17/1r; Zelenka 2008).

Telkibánya, Youth Camp (5/rf in Fig. 12)

Bodrogolaszi, Mancsalka (80/a in Fig. 12)

Erdóbénye, Szokolya (64-1-2/a in Fig. 12)

There are diatremes covered by Pannonian sediments in Bodrogköz, indicated by gravity and airborne magnetic surveys:

Apróhomok basalt (39b in Fig. 12)

Vajdácska, Várhomok (57/b in Fig. 12) 
Bodroghalom, Nyírtanya (58d in Fig. 12)

\section{Stratovolcanoes}

These volcanoes alternatively produced lava and pyroclastics (ash-flow tuff, fallen tuff). The result is a "cakelike" bedded structure, often with outwedging beds. In certain cases the same vent produced a sequence of dacitic, andesitic and rhyolitic lava and pyroclastics (Makkoshotyka Katuska, Erdőbénye Spa), lying over each other. Lava domes were also formed at these stratovolcanoes (Nagybózsva, Fekete Hill in Fig. 12).

\section{Tokaj, Nagy Hill (Kopasz Hill (Bald Hill)) (37/d in Fig. 12)}

The pyroxene dacite cone of Nagy Hill at Tokaj is characterized by massive and vesicular lava flows as well as block and ash flows with strongly oxidized lava clasts, alternating at multiple levels. There are steeply dipping, vent facies lava bodies with fluidal texture crosscutting the lava flows, partly beneath the ancient central cone, partly beneath parasitic cones. The diatremes are specific spots of different magnetism on the magnetic map. The satellite scene outlines the outcropping surface of the ancient lava flows, which are curved clockwise. The radiometric age of the repeated, mixed rhyolite-andesite lava explosions (Rózsa 1994 ) is 10.3-10.5 Ma (Pécskay et al. 1986).

Sátoraljaújhely, Fekete Hill (41/d in Fig. 12)

S from Mikóháza, Ritka Hill - Szénégetô Hill (43/rf in Fig. 12)

Makkoshotyka, Katuska Hill (21/r, 21/a in Fig. 12)

Katuska Hill at Makkoshotyka is an extrusive-effusive polyvolcano, which produced a sequence of multiply differentiated dacite-andesite-rhyolite lava. The object causes a gravity minimum, the oldest pyroxene andesite a medium magnetic anomaly, the crosscutting rhyolite complex a high total radiometric and potassium anomaly. The latter is also connected with medium $\mathrm{Hg}$ enrichment demonstrated in stream sediment samples.

Füzér, Remete Hill - Milic (1/d in Fig. 12)

Nagybózsva, Fekete Hill - Csattantyú (90/r, 90/rf in Fig. 12)

Tolcsva - Fekete Hill (73/a in Fig. 12)

Erdóbényefürdő (64/a, 64/r in Fig. 12)

\section{Single lava domes}

The single lava domes belong to no major caldera or stratovolcano, according to our present knowledge. These are morphologically and petrologically welloutlined objects, with a prominent gravity maximum in their mostly pyroclastic vicinity. Magnetite-bearing dacite and andesite bodies can cause magnetic maxima and rhyolite volcanics radiometric maxima, respectively. 
The K content of rhyolite lava bodies is medium (6-8\%), while that of rhyolite tuffs is lower $(4-5 \%)$. The Th content is regionally high $(20-30 \mathrm{ppm})$, and a pointwise hydrothermal $U$ enrichment can be observed in vent zones of rhyolite lava domes (6-10 ppm). From the centers of lava domes the lava flow facies can be followed laterally: massive, fluidal, lithophysal, spheroidal, spherolithic, perlitic (Fig. 12).

Telkibánya, Varga Hill and Nagy Valley (52/r, 52/rf in Fig. 12)

The rhyolite domes of Varga Hill and Nagy Valley at Telkibánya have nearly vertical, perlitic, fluidal texture (gray, white, red bands), and lava and debris flows with 10-30 dip branch off from these. The borehole Tb-7 crossed the covering laminar pyroxene andesite lava flow of $50 \mathrm{~m}$ thickness, reaching the underlying welded rhyolite ash-flow tuff. The domes appear as weak maxima on the total gradient map, maxima in radiometric total intensity, and Th and U content as well. The K/Ar age of the laminar, fluidal rhyolite is $13.11 \pm 0.53 \mathrm{Ma}$ (Pécskay et al. 1986).

Nagyhuta, Jakabvára Hill, Nagy-Fuló (46/r in Fig. 12)

Pusztafalu, Tolvaj Hill (3/rd in Fig. 12).

The rhyodacite lava of Tolvaj Hill at Pusztafalu broke through Sarmatian sediments. It is indicated by a relative gravity maximum of $1 \times 1 \mathrm{~km}$ in diameter. The K/Ar age of the rock is $12.6 \pm 0.5 \mathrm{Ma}$ (Pécskay et al. 1986), characterized by a large geochemical $\mathrm{Hg}$ anomaly.

Füzérkajata, Hársas Hill (4/rd in Fig. 12)

Füzérkomlós, Akasztó Hill (48/a in Fig. 12)

Füzér, Castle Hill (2/d in Fig. 12)

The dacite neck structure and morphology of Castle Hill is well visible within a $300 \times 300 \mathrm{~m}$ area with its more than $60 \mathrm{~m}$-high columns.

Füzérradvány, Korom Hill (49/r, 49/rf in Figure 12)

The maximum of the $10 \mathrm{~km}$ HP filtered gravity map at Korom Hill, Füzérradvány, indicates the 50-100 m-thick, silicified upper tuff and the sediments of the geyser basin. The geochemical anomalies $(\mathrm{Hg}, \mathrm{Au}, \mathrm{As}, \mathrm{Sb}, \mathrm{Ag})$ accompany the subsequent hydrothermal breccia zones (Fig. 9). There is a Kmetasomatized rhyolite dome in the center of the area, at Ember Rock, which was not previously recognized. The $\mathrm{K} / \mathrm{Ar}$ age of the rhyolite alteration is $12.36 \pm 0.47$ Ma (Pécskay et al. 1986). The airborne geophysical (radiometric) surveys did not cover this area. The dacite neck shows a medium magnetic anomaly, and its deep source can be recognized on the $20 \mathrm{~km}$ HP filtered gravity map. Its K/Ar age is $11.0 \pm$ 0.4 Ma (Pécskay et al. 1986; Pécskay et al. 2005). 
Areas of postvolcanic activity

The products of postvolcanic activity can be recognized around the eruption centers, partly inside the major ancient calderas and beside the vents and domes, according to geochemical anomalies. These can be placed into two major groups:

1. Epithermal polymetallic and precious metal mineralizations $(\mathrm{K}, \mathrm{Au}, \mathrm{Ag}$ enrichment).

2. Silicic and argillitic sediments of hot water basins, in some cases with As and Hg mineralization.

Some of these are quartzite veins and limnoquartzite covers exposed by erosion, easy to detect, partly by remote sensing and partly through geochemical anomalies. These formations are attached to ancient eruption centers (Fig. 9), but their K/Ar ages are always younger (Molnár and Pécskay 2002).

Telkibánya, Kánya Hill - Gyepü Hill: Au-Ag quartz veins (5/7u in Fig. 9)

Rudabányácska, Bánya Hill: Au quartz vein (41/1u in Fig. 9)

Füzérradvány, Korom-tetố: Au-Ag quartzite breccia (49/1 ru in Fig. 9)

Mád, Diós - Bomboly: gold, quartz and siderite veins (32/2u in Fig. 9)

Tolcsva, Fekete Hill - Kopaszka: Pb-Zn quartz vein (73/1u in Fig. 9)

Abaújszántó, Bányi Hill: Kuroko-type Pb, Zn, Sb (34/5u in Fig. 9)

Sárospatak, Király Hill: Hg quartzite vein (22/2u in Fig. 9)

Sárospatak, Bot-kó: limnoquartzite, bentonite $+\mathrm{Hg}$ (22/1u in Fig. 9)

Regéc, Castle Hill: limnoquartzite (15/1au in Fig. 9 )

Óhuta, Soltész Valley: quartz vein (24/1u in Fig. 9)

Erdóbénye, Ligetmajor: limnoquartzite and diatomite (28/7u in Fig. 9)

Rátka, Nemesagyag-medence: limnoquartzite, bentonite, kaolinite (33/5u in Fig. 9)

Hollóháza, Szurok Meadow: limnoquartzite, kaolinite, diatomite (5/6u in Fig. 9)

Legyesbénye, Fuló Hill: Au quartzite vein (35/22u in Fig. 9)

Szerencs, Aranka-tetô: limnoquartzite, kaolinite (35/20u in Fig. 9)

Bekecs, Nagy Hill: limnoquartzite (35/21u in Fig. 9)

Komlóska, Bolhás: quartz and calcite veins with bentonite (53/3u in Fig. 9)

Gönc, Vas Hill - Or Hill: limnoquartzite (82/1du in Fig. 9)

Eruption centers and mineral resource formation

The epithermal polymetallic and precious metal mineralizations are primarily attached to hydrothermal zones of subvolcanic intrusions, detected partly within the volcanic bodies, in their fissures, crushed zones, and partly in country rocks of the cap region, mainly in the form of veins (Telkibánya, Rudabányácska, Füzérradvány, Komlóska, Tolcsva, Sárospatak, Mád). The potassic metasomatism is characterized by adularia and feldspar formation; hydrothermal alteration by propylitic, quartz-sericite-argillitic and zeolitic zonation. In the ancient postvolcanic, hydrothermal geyser and thermal spring basins attached to the eruption centers, volcanosedimentary silica deposits, diatomite, kaolinite, illite, 
bentonite, and alunite deposits were formed (Szerencs, Rátka, Monok, Arka, Regéc, Telkibánya, Hollóháza, Füzérradvány, Sárospatak, Erdőbénye - Fig. 9). These show As, $\mathrm{Sb}, \mathrm{Hg}$ enrichment as a geochemical anomaly.

The ore and other deposit-forming processes and their products are listed at the volcanic structures, volcanic bodies and areas of postvolcanic activities above.

The magmatic rocks solidified from lavas; pyroclastics of the volcanoes are used as building stone. The lava plugs of the subvolcanic rock bodies and the diatremes provide large resources; they contain subsurface lamination or columnar joints, formed perpendicular to the cooling surface by slow cooling. In the shallow levels the rocks are glassy and fine-grained, in the depth becoming gradually coarse crystalline, partly diorite porphyry. These rocks are exploited in quarries (Kopasz Hill 33/a at Tállya, Mulató Hill 61/a at Erdóbénye).

The thick andesitic or dacitic lava flows of the stratovolcanoes are fine-grained and high-strength materials as well, but the inhomogeneous material of tuff, agglomerate and debris flows between the lava beds produced by periodical volcanic activity are inappropriate for exploitation (Nagy Hill 37/d at Tokaj).

The central zones of volcanic fissures are good-quality but low-volume building stone occurrences (Gombos 78/a at Hercegkút, Páncél Hill 78/2a and Szent Vince Hill 78/3a at Sárospatak).

The single rhyolite extrusive domes produced perlitic lava bodies and in some sites lava flows, mainly at underwater extrusions as pumiceous lava facies. The extrusive perlite with columnar joints of Pálháza (17/1r), the pitchstone and pumice breccia of Páskatető (47/r) at Nagybózsva and the perlitic bodies of the Ósva Valley between Telkibánya and Pálháza belong to this type. This rock material is appropriate for insulation purposes. The exploitation is restricted by natural protection of the occurrence areas.

The acidic volcanic glass (fallen ash) produced loose pumiceous tuff (pumicite), which can also swell, but its strength is low (Szegi).

The facies of ignimbritic flow tuff, deposited mostly in water, was transformed into zeolite (clinoptilolite, mordenite) in the matrix and on the edges of pumice lapilli as well, forming economic zeolitic rhyolite tuff deposits (Rátka-Koldu, Bodrogkeresztúr, Mezozombor, Mád).

\section{Identified tectonic lineaments of the Tokaj Mountains}

Based on satellite imagery covering the entire range (Landsat, radar SRTM), on stereo-aerial photography and on geophysical gravity and airborne magnetic surveys the major tectonic zones drawn in Figure 13 were constructed. These are partly visible on the surface, partly buried structures detected by geophysical methods; however, their strike can be identified unambiguously. The short characteristics of the structures are listed in Table 2. 


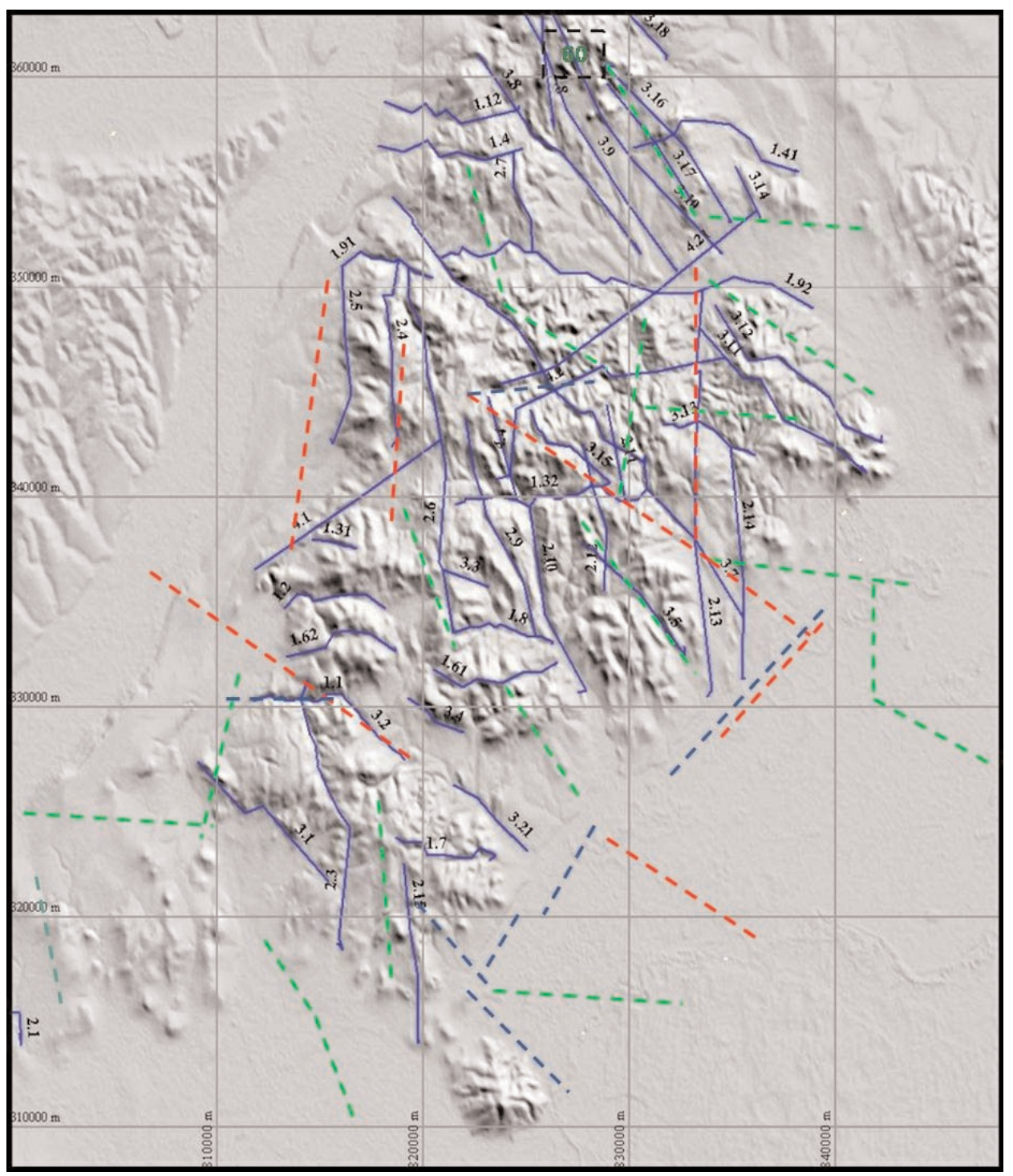

Fig. 13

Structural scheme based on SRTM topography (blue lines), gravimetric (green lines) and magnetic (red lines) surveys (T. Zelenka, J. Kiss). E-W striking: strike-slip and normal faults with downthrow to S (e.g. Bózsva Creek Valley 1.92).

N-S (NNW-SSE) striking: volcanotectonic extension faults along the boundary of volcanics and at veins (e.g. Telkibánya 2.7).

NW-SE striking: faults with large-scale downthrow along the boundary of the mountains, in different rock masses and volcanic fissures.

NE-SW (NNE-SSW) striking: faults with small-scale downthrow or strike-slip sense on the boundary of the mountains and at different rock masses (e.g. Hernád Valley, Kemence Creek). 
Table 2

Tectonic structures of the Tokaj Mountains

\begin{tabular}{|c|c|c|c|c|c|c|c|c|c|}
\hline Tectonic structure, site & Structure type & $\begin{array}{l}\text { 하 } \\
\text { 응 } \\
\stackrel{0}{0}\end{array}$ & 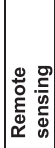 & 䇉 & 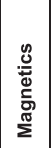 & 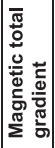 & 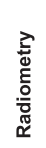 & 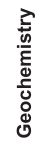 & 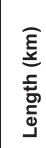 \\
\hline \multicolumn{10}{|l|}{ 1. E-W (ESE-WNW) structures } \\
\hline 1.1 Abaújkér, Aranyos Valley & zigzag fault & + & + & + & & & + & & 4.76 \\
\hline 1.2 Boldogköváralja, Tekeres Valley & zigzag fault, $\mathrm{S}$ downthrow & & + & + & + & & + & & 5.74 \\
\hline 1.3 Óhuta - Újhuta, Hutai Valley & zigzag fault, $\mathrm{S}$ downthrow & + & + & + & + & + & & + & 10.6 \\
\hline 1.4 Pányok, Hasdád Valley & zigzag fault & + & & + & & & & + & 7.78 \\
\hline 1.5 Erdőbénye, Faragványos & zigzag fault, $\mathrm{S}$ downthrow & + & & + & & & & + & 6.78 \\
\hline 1.6 Erdőhorváti, Szokolya & zigzag fault, $\mathrm{S}$ downthrow & & + & + & + & & & + & 6.28 \\
\hline 1.7 Szegilong, Cigány Hill - Meszes & formation boundary, fault & + & + & + & & + & & & 6.05 \\
\hline 1.8 Erdöhorváti, Hárskút Tolcsva Creek & formation boundary & + & + & + & & & & & 5.3 \\
\hline 1.9 Nagybózsva, Bózsva Valley & formation boundary, fault, $\mathrm{N}$ downthrow & + & + & + & + & + & + & & 5.03 \\
\hline 1.10 Újhuta, Szpalanyica Valley & formation boundary & + & & + & & & + & + & \\
\hline 1.11 Nagyhuta, Hallás Valley & formation boundary, zigzag fault & + & & + & & & + & + & \\
\hline 1.12 Kéked, Hosszú Valley & formation boundary & + & + & + & & & + & & \\
\hline \multicolumn{10}{|l|}{ 2. N-S (NNW-SSE) structures } \\
\hline 2.1 Monok, Nyires & fault, W downthrow & & + & + & + & & & & 9.87 \\
\hline 2.2 Monok, Nagyrépás - Majos Hill & \begin{tabular}{|l|} 
volcanotectonic \\
\end{tabular} & & & & + & + & + & & 5.35 \\
\hline 2.3 Tállya, Hideg Valley & & + & + & + & + & + & & & 14.8 \\
\hline 2.4 Gönc (Amádévár), Kis Creek & volcanotectonic & & + & + & & & & & 9.57 \\
\hline $\begin{array}{l}2.5 \text { Gönc - Hejce - Fony, W border of } \\
\text { the Mts. }\end{array}$ & formation boundary, fault, W downthrow & + & + & + & + & + & + & + & 9.1 \\
\hline 2.6 Telkibánya - Regéc, Nagy Creek & $\begin{array}{l}\text { volcanotectonic, formation boundary, } \\
\text { quartz veins }\end{array}$ & + & + & + & & & + & + & 17.8 \\
\hline $\begin{array}{l}\text { 2.7 Telkibánya, Kánya Hill Veresvíz } \\
\text { Valley }\end{array}$ & $\begin{array}{l}\text { volcanotectonic, fault, E downthrow, ore } \\
\text { vein }\end{array}$ & + & + & + & & & & + & 5.18 \\
\hline 2.8 Füzér, Bisó Creek & formation boundary, fault, $\mathrm{W}$ downthrow & + & + & + & & & & & 7.38 \\
\hline 2.9 Erdöhorváti, Tolcsva Creek & & + & + & + & & + & & + & 13.9 \\
\hline $\begin{array}{l}2.10 \text { Erdöhorváti - Középhuta, Egres } \\
\text { Creek }\end{array}$ & formation boundary & + & + & + & + & & & + & 15.8 \\
\hline 2.11 Komlóska & formation boundary, fault, ore vein & + & & + & & & + & & 4.67 \\
\hline 2.12 Újhuta, Komlóska Creek & & & & + & & & & & 7.17 \\
\hline 2.13 Kovácsvágás, Hosszú Creek & formation boundary, fault, $\mathrm{W}$ downthrow & + & + & + & & + & & & 20.0 \\
\hline $\begin{array}{l}\text { 2.14 Hercegkút - Sárospatak, } \\
\text { Hercegkút Creek }\end{array}$ & $\begin{array}{l}\text { formation boundary } \\
\text { fault, } W \text { downthrow, andesite dykes }\end{array}$ & + & + & + & & + & + & & 11.3 \\
\hline 2.15 Bodrogkeresztúr Kakas-nyereg & $\begin{array}{l}\text { formation boundary, rhyolite, andesite } \\
\text { dyke }\end{array}$ & + & + & + & & & + & & 8.76 \\
\hline 2.16 Sima - Baskó, Sás Creek & formation boundary & + & + & + & + & & & & \\
\hline \multicolumn{10}{|l|}{ 3. NW-SE structures } \\
\hline 3.1 Abaújszántó, Krakó Hill - Sátor Hill & formation boundary, fault, SW downthrow & + & + & + & & + & & + & 9.14 \\
\hline $\begin{array}{llll}3.2 & \text { Erdöbénye, Aranyos Creek - } \\
\text { Mélyviz } & & & \\
\end{array}$ & formation boundary, fault, E downthrow & + & + & + & + & & + & & 4.37 \\
\hline 3.3 Erdőhorváti, Hajagos Valley & formation boundary & + & + & + & + & & + & & 2.35 \\
\hline 3.4 Erdőbénye, Nagy-Mondoha & formation boundary, fault, SE dow & + & + & + & + & + & + & & 3.35 \\
\hline 3.5 Komlóska - Tolcsva, Hideg Valley & formation boundary & + & + & + & + & + & + & & 7.84 \\
\hline $\begin{array}{l}3.6 \text { Makkoshotyka, Hotyka Creek } \\
\text { Valley }\end{array}$ & formation boundary & + & + & + & + & + & + & + & \\
\hline 3.7 Telkibánya, Ósva Valley & strike-slip fault & + & & + & + & + & + & & 28.2 \\
\hline 3.8 Nyíri, Nyíri Creek Valley & formation boundary, strike-slip fault & + & + & + & & & & & 13.1 \\
\hline 3.9 Hollóháza, Török Creek Valley & formation boundary, fault & + & + & + & + & + & & & 14.7 \\
\hline 3.10 Füzér - Füzérradvány, Bisó Creek & formation boundary, fault, SW downthrow & + & + & + & + & & & & 14.4 \\
\hline 3.11 Kovácsvágás, Gyékényes-árok & formation boundary, fault, SW downthrow & & & + & + & + & & + & 11.4 \\
\hline $\begin{array}{l}3.12 \text { Rudabányácska, Magas Creek - } \\
\text { Hármaskúti Valley }\end{array}$ & & & & + & & & + & + & 11.6 \\
\hline 3.13 Vágáshuta, Fehér Creek Valley & forma & + & + & + & + & + & + & & 5.11 \\
\hline 3.14 Vilyvitány, Gödölye-árok & $\begin{array}{l}\text { formation boundary, fault, SW downthrow, } \\
\text { breccia, dykes }\end{array}$ & + & + & + & + & + & & & 2.82 \\
\hline 3.15 Óhuta, Nagy-Oldal Valley & formation boundary & + & + & + & + & & & & 5.89 \\
\hline 3.16 Füzérkajata, Hársas - Korom Hill & formation boundary, fault, NE downthrow & + & + & + & + & & & + & 10.3 \\
\hline 3.17 Füzér, Remete Hill - Ör Hill & formation boundary, fault, SW downthrow & + & + & + & + & & & + & 7.14 \\
\hline \multicolumn{10}{|l|}{ 4. NE-SW (NNE-SSW) structures } \\
\hline 4.1 Arka, Boldogkőváralja Creek & formation boundary, zigzag & + & + & + & & & & + & 11.0 \\
\hline 4.2 Kökapu - Pálháza, Kemence Creek & strike-slip fault & & + & + & & + & & & 11.1 \\
\hline 4.3 Nagybózsva, Senyő Valley & tion boundary & + & + & + & & + & & & \\
\hline 4.4 Kökapu, Ördög Valley & formation boundary & + & + & + & + & + & & & 3.9 \\
\hline
\end{tabular}




\section{Summary}

The nearly N-S striking Tokaj Mountains are situated in the northeastern part of the Miocene ALCAPA microplate, between the Pannonian Basin and the inner side of the Carpathian Arc. The range extends into the Slanské Mts in Slovakia. The mountains were formed in the Middle and Late Miocene during subduction, with the calc-alkaline bimodal, intermediate and felsic volcanism of the ancient inner island arc. The mountains are bordered by tectonic zones in a triangle shape. The basement and the Miocene volcanics become thicker to the $\mathrm{W}$, indicating back-arc basin character. In the northeastern part of the Tokaj Mts there are Lower Paleozoic and Proterozoic metamorphic rocks at surface; in the southeastern part of the basement Mesozoic, predominantly carbonate rocks were encountered in boreholes. In the $\mathrm{W}$ shale is indicated by xenoliths in pyroclastics. The eastern part of the mountains comprises Upper Badenian Sarmatian shallow-lagoon sediments and nearly $1000 \mathrm{~m}$ thick pyroclastics (produced by phreatomagmatic eruptions) with dacite subvolcanic bodies. In the western part of the Tokaj Mts three volcanic cycles produced nearly $2500 \mathrm{~m}$ thick successions in the descending lagoon:

1. Upper Badenian (14-15 Ma) rhyolite-dacite pyroclastic flows, then subaqueous peperitic, hyaloclastitic, stratovolcanic andesite with lava beds and rhyodacite subvolcanoes.

2. Lower Sarmatian (12-13 Ma) large volumes of phreatomagmatic ignimbrite flows from rhyolite calderae, fallen pyroclastics and single rhyolite domes. In the central zone of the mountains there are several large andesite stratovolcanoes and subvolcanoes, with attached hydrothermal epithermal precious metal mineralization and less well-known $\mathrm{Pb}-\mathrm{Zn}$ enrichment.

3. At the Sarmatian - Pannonian boundary (10-11 Ma): phreatomagmatic rhyolitic ignimbrites, rhyolite domes and andesitic-dacitic stratovolcanoes; finally (9-10 Ma) olivine andesite domes, dykes and calc-alkaline olivine basalt shield volcanoes as final products.

Since then, on average $200-300 \mathrm{~m}$ of material was eroded from the uplifted area.

\section{Acknowledgements}

The authors are grateful to the Hungarian Scientific Research Fund OTKA for supporting the T-022769: "Paleovolcanic reconstruction of the Tokaj Mountains" project, and for the supporting studies of other researchers participating in this project: László Vértesy (ELGI) carried out geophysical, István Horváth (MÁFI) geochemical, Emó Szalay (ELGI) paleomagnetic measurements, Zoltán Pécskay (ATOMKI) radiometric age determinations. Beyond these, we also appreciate and utilized the critical remarks regarding this paper from Prof. János Földessy (University of Miskolc) and the able editorial assistance of Péter Fuchs and Norbert Németh (University of Miskolc). 


\section{References}

Beudant, F. S. 1822: Voyage mineralogique et geologique en Hongrie pendant l'année 1818. (Geological and mineralogical field trip in Hungary during 1818), Vols 1-3. + Atlas - Verdiére, Paris (In French.)

Cas, R.A.F, J.V. Wright 1988: Volcanic Successions - Modern and Ancient. - Unwin Hyman, London, $528 \mathrm{p}$.

Gulyás, Â., J. Kiss, T. Zelenka 2000: Kálimetaszomatizált területek kimutatása és lehatárolása légi geofizikai és földtani módszerekkel (Detection and definition of areas affected by potassic metasomatism by airborne geophysics and geologic methods). - Földtani Kutatás, 37/3, pp. 19-23. (In Hungarian.)

Gyarmati, P. 1977a: A Tokaji-hegység szerkezeti-vulkanotektonikai vázlata (térkép) (Structuralvolcanotectonic sketch of the Tokaj Mts [map]). - Supplement to the MÁFI Évkönyv 58, (Hungarian - English).

Gyarmati, P. 1977b: A Tokaji-hegység intermedier vulkanizmusa (Intermediate volcanism in the Tokaj Mts). - MÁFI Évkönyv 58, pp. 1-195. (Hungarian - English)

Gyarmati, P. 1997: A Tokaji-hegység (The Tokaj Mts). - In: Pannon Enciklopédia. - Kertek 2000 Kiadó, Budapest, pp. 387-391. (In Hungarian.)

Gyarmati, P., T. Zelenka 1968a: A Tokaji-hegység földtani térképe. 25 000-es sorozat. Mád (Geological map of the Tokaj Mts, 1:25 000 scale series, Mád sheet). - MÁFI, Budapest. (In Hungarian.)

Gyarmati, P., T. Zelenka 1968b: Magyarázó a Tokaji-hegység földtani térképéhez. 25 000-es sorozat. Mád (Explanatory volume to the geological map of the Tokaj Mts, 1:25 000 scale series, Mád sheet). - MÁFI, Budapest, 70 p. (In Hungarian.)

Gyarmati, P., E. Perlaki, L. Pentelényi 1977: A Tokaji hegység földtani térképe (Geological map of the Tokaj Mts). - Supplement to the MÁFI Évkönyv 58, (Hungarian - English).

Gyarmati, P., T. Zelenka 2000: A Tokaji-hegység paleovulkáni újraértékelése (Paleovolcanic reinterpretation of the Tokaj Mts). - manuscript (In Hungarian.)

Hartikainen, A., I. Horváth, L. Ódor, L. Ó Kovács, J. Csongrádi 1992: Regional multimedia geochemical exploration for Au in the Tokaj Mountains, northeast Hungary. - Applied Geochemistry, 7/6, pp. 533-547.

Hartikainen, A., I. Horváth, L. Ódor, L. Ó. Kovács, U. Fügedi 1993: Regional geochemical survey of the Tokaj Mountains, northeast Hungary. - Tutkimusraportti (Report of Investigation) 120, Geological Survey of Finland, Espoo, 32 p.

Hegedûs, E., K. Posgay, T. Bodoky, T. Fancsik, A. Cs. Kovács, R. Csabafi and Celebration Working Group, 2002: 3D refraction tomographic images from the Mátra-Zemplén region. - Geologica Carpathica 53 Special CD Issue: Proceedings of XVII. Congress of Carpathian-Balkan Geological Association Bratislava, September 1st - 4th 2002.

Hoffer, A. 1928: Néhány szó a Tokaji-hegység eruptivumainak településéhez (Some remarks on the stratigraphy of eruptive rocks in the Tokaj Mts). - Földtani Közlöny, 58, pp. 127-130. (In Hungarian.)

Hoffer, A. 1937: A Szerencsi-sziget földtani viszonyai (Geological features of the Szerencs Inselberg). - A debreceni Tud. Egy. Ásvány-földtani Intézetének Közl., 8 (Tisia 2), Debrecen (In Hungarian.)

Horváth, J., T. Fegyvári, T. Zelenka 1989: Paleovolcanic structures in the North-Tokaj Mountains interpreted on the basis of the satellite imagery and aerial photography. - Acta Geologica Hungarica, 32/1-2, pp. 47-51.

Horváth, J., T. Zelenka 1997: A telkibányai nemesfém-ércesedés legújabb bányaföldtani adatai és értékelése (The latest data on the Telkibánya precious metal mineralization and their evaulation). - Földtani Közlöny, 127/3-4, pp. 405-430. (In Hungarian.)

Ilkeyné Perlaki, E. 1967: Magyarázó a Tokaji-hegység földtani térképéhez, 25 000-es sorozat. Gönc (Explanatory volume to the geological map of the Tokaj Mts, 1:25 000 scale series, Gönc sheet). - MÁFI, Budapest, 48-63 p. (In Hungarian.) 
Ilkeyné Perlaki, E. 1978: Magyarázó a Tokaji-hegység földtani térképéhez, 25 000-es sorozat. Nyíri (Explanatory volume to the geological map of the Tokaj Mts, 1:25 000 scale series, Nyíri sheet). - MÁFI, Budapest, 55 p. (In Hungarian.)

Jugovics, L. 1962: Kőbányászatunk fejlesztése céljából 1948-1960. évek alatt végzett geológiai kutatások gyakorlati eredményei (Practical results of geological explorations for development of quarrying in the period 1948-1960). - Építőanyag 14/3-4, pp. 116-120, 142-148. (In Hungarian.)

Kiss, J., Á. Gulyás 1998: Jelentés a Légigeofizikai és Távérzékelési Laboratórium 1998. évi feladatairól (Report on the tasks of the Aerogeophysical and Remote Sensing Laboratory in 1998). Manuscript, ELGI archive (In Hungarian.)

Kiss, J., E. Prácser 2000: Szelvénymenti gravitációs és légimágneses feldolgozások a Tokaji-hegység területén (Interpreted gravity and airborne magnetic sections in the Tokaj Mts). - Földtani Kutatás, 37/4, pp. 18-24. (In Hungarian.)

Kiss, J., T. Zelenka 2009: Geological features, geophysical measurements and interpretation at the Telkibánya research area. - Publications of the University of Miskolc, Series A, Mining 78, pp. 97-113.

Kulcsár, L., I. Bartha 1971: Kőzettani vizsgálatok az erdőbényei Mulatóhegy-Barnamáj lakkolitján (Petrographical studies on the Mulatóhegy-Barnamáj laccolith near Erdóbénye village). - Acta Geogr. Debrecina, 15-16. pp. 39-72. (In Hungarian.)

Lengyel, E. 1959: Földtani és kôzettani megfigyelések a Tokaji-hegységben (Geological and petrological observations in the Tokaj Mts). - Földtani Közlöny, 89/4, pp. 381-392. (In Hungarian.)

Liffa, A. 1940: Boldogkőváralja és környékének geológiai viszonyai (Geological features of the vicinity of Boldogkőváralja). - MÁFI Évi Jel. 1933-35, pp. 1337-1411. (In Hungarian.)

Liffa, A. 1953: Telkibánya környékének földtana és kőzettana (Geology and petrology of the Telkibánya area). - MÁFI Évkönyv, 41/3, pp. 1-78. (In Hungarian.)

Molnár, F., T. Zelenka 1995: Fluid inclusion characteristics and paleothermal structure of the adularia-sericite type epithermal deposite at Telkibánya, Tokaj Mts Northeast Hungary. Geologica Carpathica, 46/4, pp. 205-215.

Molnár, F, T. Zelenka, E. Mátyás, Z. Pécskay, B. Bajnóczi, J. Kiss, I. Horváth 1999: Epithermal mineralization of the Tokaj Mountains, Northeast Hungary: Shallow levels of low-sulfidation type systems. - In: Molnár, F., J. Lexa, J.W. Hedenquist (Eds): Epithermal mineralization of the Western Carpathians. - Guidebook Series 31, Guidebook prepared for the Society of Economic Geologists Field Conference, 4-13. September 1999, pp. 109-153.

Molnár, F, Z. Pécskay 2002: Relationships between volcanism and hydrothermal activity in the Tokaj Mountains, Northeast Hungary, based on K-Ar ages. - Geologica Carpathica, 53/5, pp. 303-314.

Nairn, A.E.M., J. Negendank, G. Pantó 1971: Palaeomagnetic investigations of the Tertiary and Quaternary igneous rocks: IV. The Tertiary volcanic rocks of the Tokaji Mountains, Hungary. Geologische Rundschau, 60/2, pp. 727-743.

Pantó, G. 1963: Ignimbrites of Hungary with regard to their genetics and classification. - Bulletin of Volcanology, 25/1, pp. 175-181.

Pantó, G. 1967: A plutói és vulkáni kôzetképződés határkérdései (Borderline problems of plutonic and volcanic rock formation). - MTA X. Oszt. Közl. 1, pp. 67-78. (In Hungarian.)

Pálfy, M. 1927: Adatok a Tokaji hegység harmadkori erupcióinak korviszonyaihoz (Data to the age of the Tertiary eruptions in the Tokaj Mts). - Földtani Közlöny, 57, pp. 67-71. (In Hungarian.)

Pentelényi, L. 1967: Magyarázó a Tokaji-hegység földtani térképéhez. 25 000-es sorozat. Erdőbénye (Explanatory volume to the geological map of the Tokaj Mts, 1:25 000 scale series, Erdóbénye sheet). - MÁFI, Budapest, 52 p. (In Hungarian.)

Pécskay, Z., K. Balogh, V. Széky-Fux, P. Gyarmati 1986: Geochronological investigations on the Neogene volcanism of the Tokaj Mountains. - Geologicky Zborník - Geologica Carpathica, 37/5, pp. 635-655. 
Pécskay, Z., J. Lexa, A. Szakács, K. Balogh, I. Seghedi, V. Konecny, E. Kovács, E. Márton, M., Kaliciak, V. Széky-Fux, T. Póka, P. Gyarmati, O. Edelstein, E. Rosu, B. Zec 1995: Space and time distribution of Neogene-Quaternary volcanism in the Carpatho-Pannonian Region. - Acta Vulcanologica, 7/2, pp. 15-28.

Pécskay, Z., F. Molnár, T. Itaya, T. Zelenka 2005: Geology and K-Ar geochronology of illite from the clay deposit at Füzérradvány, Tokaj Mts, Hungary. - Acta Mineralogica-Petrographica, 46, pp. 1-7.

Richthofen, F. 1861: Studien aus dem ungarisch-siebenbürgischen Trachytgebirge (Observations in the Hungarian-Transylvanian trachyte mountains). - Jahrbuch der Geologischen Reichsanstalt 11, pp. 153-278. (In German)

Rozlozsnik, P. 1937: A Tokaj-Hegyalja DNY-i részének s a vele dél felől határos sík terület földtani viszonyai (Geological features of the SW part of the Tokaj-Hegyalja and the surrounding plain to S). - MÁFI Évi Jel. 1929-32, pp. 329-364. (In Hungarian.)

Rózsa, P. 1994: The dacite flows of the Miocene Tokaj-Nagyhegy stratovolcano: an example of magma mixing. - Geol. Carpathica, 45/3, pp. 139-144.

Szabó, J. 1867: Tokaj-Hegyalja és környékének földtani viszonyai (Geological features of TokajHegyalja and its surroundings). - Mathematikai és Természettudományi Közlemények, 4, pp. 226-303. (In Hungarian.)

Szádeczky, Gy. 1897: A Sátoraljaújhelytől ÉNY-ra, Rudabányácska és Kovácsvágás közé eső terület geológiai és kőzettani tekintetben (The area NW from Sátoraljaújhely, between Rudabányácska and Kovácsvágás from a geological-petrographical point of view). - Földtani Közlöny, 27, pp. 273-326. (In Hungarian.)

Szalay, E., Z. Pécskay, T. Zelenka 2007: A Tokaji-hegységi miocén vulkanitok paleomágneses és K/Ar koradatainak területi, időbeli változásai a földtani képződményekben (Spatial and temporal variance of paleomagnetic and K/Ar age data in the Miocene volcanics of the Tokaj Mts). manuscript (In Hungarian.)

Székyné Fux, V. 1970: Telkibánya ércesedése és kárpáti kapcsolatai (The mineralization of Telkibánya and its connections to the Carpathians). - Akadémiai Kiadó, Budapest, 266 p. (In Hungarian.)

Telegdi Róth, K. 1935: Az 1934. évben Erdôbénye környékén végzett geológiai felvételrôl (On the geological record at Erdőbénye in 1934). - Manuscript, MÁFI archive (In Hungarian.)

Wolf, H. 1869: Erläuterungen zu den geologischen Karten der Umgebung von Hajdunánás, Tokaj und Sátoraljaújhely (Explanations to the geological maps of the vicinity of Hajdunánás, Tokaj and Sátoraljaújhely). - Jahrbuch der Geologischen Reichsanstalt 19, pp. 235-264. (In German.)

Zelenka, T. 1964: A "Szerencsi-öböl" szarmata tufaszintjei és fáciesei (Sarmatian tuff horizons and facies of the 'Szerencs Embayment'). - Földtani Közlöny, 94/1, pp. 33-52. (In Hungarian.)

Zelenka, T. 1967: Veränderungen vulkanischer Gläser aufgrund mikroskopischer Untersuchungen (Alteration of volcanic glasses as observed under the microscope). - Acta Geologica Hungarica, 11/4, pp. 437-451. (In German)

Zelenka, T. 1997: Paleovulkáni szerkezetek felismerése és azonosítása ûrfotók alapján Magyarországi példákon (Recognition and identification of paleovolcanic structures on satellite imagery, case studies from Hungary). - VII. Földfelszíni és meteorológiai megfigyelések a Világúrből szeminárium, Magyar Asztronautikai Társaság, pp. 76-82. (In Hungarian.)

Zelenka, T. 1998: Recognition and identification of paleovolcanic structures in Hungary by space photographs. - Carp.-Balkan. Geol. Ass. XVI. Congress, p. 649.

Zelenka, T. 2000: A Tokaji-hegység vulkáni szerkezeteinek távérzékeléses (ûr- és légifotó) vizsgálatai (Remote sensing studies (satellite imagery and airborne photography) of the volcanic structures in the Tokaj Mts). - Földtani Kutatás, 37/4, pp. 13-17. (In Hungarian.)

Zelenka, T. 2008: A pálházai perlitelőfordulás földtana (Geology of the perlites in the Pálháza region). - 6th International Perlite Conference and Exhibition, Scientific Society of the Silicate Industry, pp. 51-76. (In Hungarian.)

Zelenka, T, P. Gyarmati, J. Kiss, L. Vértesy, I. Horváth, Z. Pécskay, E. Szalay 2007: A Tokaji-hegység paleovulkáni rekonstrukciója (Paleovolcanic reconstruction of the Tokaj Mts). - Manuscript (MTA Geochemistry Comittee Report), 55 p. (In Hungarian.) 